\title{
SORTING AND DECENTRALIZED PRICE COMPETITION
}

\author{
By JAN EECKHOUT AND PHILIPP KIRCHER ${ }^{1}$
}

\begin{abstract}
We investigate the role of search frictions in markets with price competition and how it leads to sorting of heterogeneous agents. There are two aspects of value creation: the match value when two agents actually trade and the probability of trading governed by the search technology. We show that positive assortative matching obtains when complementarities in the former outweigh complementarities in the latter. This happens if and only if the match-value function is root-supermodular, that is, its $n$th root is supermodular, where $n$ reflects the elasticity of substitution of the search technology. This condition is weaker than the condition required for positive assortative matching in markets with random search.
\end{abstract}

KEYwORDS: Competitive search equilibrium, directed search, two-sided matching, decentralized price competition, complementarity, root-supermodularity, sorting.

\section{INTRODUCTION}

WE ADDRESS THE ROLE OF SEARCH FRICTIONS in the classic assignment problem when there is price competition. We are interested in a simple condition for positive assortative matching (PAM) that exposes the different forces that induce high types to trade with other high types. In the neoclassical benchmark (Becker (1973), Rosen (1974)), there is full information about prices and types, and markets clear perfectly. Supermodularity of the match value then induces PAM. At the other extreme, Shimer and Smith (2000) assumed that there are random search frictions, and agents cannot observe prices and types until after they meet. They derived a set of conditions that ensure PAM and that jointly imply that the match value is log-supermodular. In this paper, we consider a world with search frictions, yet there is information about prices and types. This circumvents the feature of the random search model that agents necessarily meet many trading partners that they would rather have avoided. Heterogeneous sellers compete in prices for buyers, and we find that sorting is driven by a simple efficiency trade-off between the gains from better match values and the losses due to no trade. The former are captured by complementarities in the match value, which have to offset complementarities in the search technology as measured by the elasticity of substitution. This economic trade-off establishes that PAM occurs for all type distributions if and only if the match value is root-supermodular, that is, its $n$th root is supermodular where $n$ depends on the elasticity of substitution of the search technology. This condition

\footnotetext{
${ }^{1}$ The paper initially circulated under the title "The Sorting Effect of Price Competition." We would like to thank numerous colleagues and seminar participants for insightful discussions and comments. We greatly benefited from comments by Ken Burdett, John Kennan, Stephan Lauermann, Benny Moldovanu, Michael Peters, Andrew Postlewaite, Shouyong Shi, Robert Shimer, and Randy Wright. Kircher gratefully acknowledges support from the National Science Foundation, Grant SES-0752076, and Eeckhout acknowledges support from the ERC, Grant 208068.
} 
is weaker than log-supermodularity and has a transparent economic interpretation.

The key ingredients of our model are diversity, market frictions, and price competition. Diversity is the hallmark of economic exchange. People have different preferences over goods and are endowed with diverse talents. Such diverse tastes and endowments lead to different market prices that are driven by the supply and demand of each variety. Spatially differentiated goods like houses, for example, are priced depending on the characteristics of the occupants, location, and the dwelling itself. Assets in the stock market are differentiated depending on many characteristics, most notably mean and variance. In labor markets, salaries vary substantially depending on the experience and skill of the worker and on the productivity and safety of the job. While centralized price setting (see Rosen (2002), for an overview) adequately captures environments such as the stock market, in many other environments trading is decentralized and frictions are nonnegligible. In the labor market, for example, unemployment is a natural feature; in the housing market, several months delay in finding a buyer is usual.

To captures these features, we consider a decentralized market framework with search frictions, yet with price competition. This framework is known as directed search or competitive search. Sellers have one unit for sale and buyers want to buy one unit. Think of "locations" or "submarkets" indexed by the quality of the product and the trading price. Sellers of a particular quality choose the location with the price they want to obtain. Buyers observe the sellers at the various locations and decide at which location they would like to trade, that is, which quality-price combination to seek. At each location there remain search frictions that prevent perfect trade: When the ratio of buyers to sellers at a location is high, then the probability of trade is high for the sellers and low for the buyers. Observe that the location metaphor is used for simplicity but is not crucial (e.g., in Peters (1991, 1997a) buyers choose an individual seller with the desired quality-price announcement, but sometimes multiple buyers choose the same seller and not all can trade). Prices guide the trading decisions just like in the Walrasian model of Becker (1973) and Rosen (1974), only now the possibility that a person cannot trade remains an equilibrium feature that is taken into account in the price setting. One novelty of our setting relative to the earlier directed search literature is that it is designed to handle rich (continuous) type distributions on both sides of the market.

We identify the economic forces that drive the sorting pattern, and provide a necessary and sufficient condition on the strength of supermodularity that ensures positive assortative matching. The key economic insight is that the creation of value can be decomposed into two sources: the complementarity in the match value upon trading and the complementarity in the search technology. In the Walrasian framework, only the first source is present. When both are present, they trade off against each other: the first leads toward positive assortative matching; the second leads toward negative assortative matching. 
If the former outweighs the latter, positive assortative matching obtains. We can summarize the necessary and sufficient condition required for PAM by root-supermodularity of the match-value function, that is, the $n$th root of the match-value function is supermodular. The magnitude of $n$ is determined by the upper bound of the elasticity of substitution of the search technology. Similarly, match values that are nowhere $n$-root-supermodular lead to negative assortative matching (NAM), where $n$ now denotes the lower bound of the elasticity of substitution in the search technology.

The economic intuition of this trade-off between frictions and complementarities in match values is transparent in terms of the fundamentals of the economy. In the absence of any complementarities, sorting is not important for the creation of match value. The key aspect is "trading security," that is, to ensure trade and avoid frictions. High-type buyers would like to trade where few other buyers attempt to trade. This allows them to secure trade with high probability and they are willing to pay for this. While sellers know that they might be idle if they attract few buyers on average, some are willing to do this at a high enough price. The low-type sellers are those who find it optimal to provide this trading security, as their opportunity cost of not trading is lowest. This results in negative assortative matching: high-type buyers match with low-type sellers. In the directed search literature, Shi (2001) was the first to highlight for a specific search technology that supermodularity is not enough to ensure positive assortative matching. Here, we address in a general context the extent of the complementarities required for positive assortative matching and we isolate the economic forces that govern such sorting. ${ }^{2}$

How much supermodularity is needed-how fast marginal output changes across different matched types-depends on how fast the probability of matching changes when moving across different types with different buyer-to-seller ratios. The change in the matching probability is captured by the elasticity of substitution of the search technology. The elasticity of substitution measures how many more matches are created as the ratio of buyers to sellers increases. If it is high, then matching rates are very sensitive to the buyer-seller ratio and submarkets with lots of low-type sellers make it easy for the high-type buyers to trade, while submarkets with lots of low-type buyers make it easy for the high seller types to trade. The "trading-security" motive is important since the gains from negative sorting are large, and positive sorting only arises if the match value improves substantially when high types trade with high types rather than low types. If the elasticity of substitution in the search technology is low, then it is difficult to generate additional matches for the high types and even moderate strength of the match value motive will offset the tendency to seek trading security.

The exact level of supermodularity required for positive sorting can be expressed by requiring a concave transformation of the match value to be su-

\footnotetext{
${ }^{2}$ We relate our findings to Shi's (2001) insight in greater detail in Section 6.
} 
permodular. In particular, it can be summarized by the (relative) Arrow-Pratt measure of the transform, which has to be as large as the elasticity of substitution of the search technology. The latter is in the unit interval, so the associated transform is the $n$th root, where $n$ depends on the exact magnitude of the elasticity of substitution. The root-supermodularity condition therefore neatly summarizes the trade-off between complementarity in match value and the elasticity of substitution of the search technology.

For PAM our condition is weaker than log-supermodularity required in random search models such as Shimer and Smith $(2000)^{3}$ and Smith (2006). The key difference is that our framework allows agents to seek the quality and price they desire. This leads to a rather simple and straightforward condition for sorting. It requires a lower degree of complementarity in the match value to overcome the search frictions. Only when the search technology approaches perfect substitutability is log-supermodularity needed. Our condition for positive assortative matching therefore falls in between those for frictionless trade of Becker and random search. Yet, when it comes to negative assortative matching, our results differ substantially. Match values that are nowhere $n$-root-supermodular induce negative sorting. In particular, this is the case for any weakly submodular match-value function. If the matching technology never approaches perfect complementarity (this excludes the urn-ball search technology), then there are strictly supermodular match-value functions such that negative sorting arises for any distribution of types. To our knowledge, this is new in the literature on sorting with or without frictions. In comparison, negative assortative matching obtains only under stronger conditions both in the frictionless case (strict submodularity) and with random search (logsubmodularity).

Our requirement of root-supermodularity is necessary and sufficient to ensure positive assortative matching if we allow for any distribution of types. It is binding when the buyer-seller ratio in some market induces the highest possible elasticity of substitution of the search technology. For some distributions, this is not a binding restriction, and in this case there are match value functions with less complementarity that nonetheless induce positive assortative matching. In that sense, our condition is one of weak necessity. Likewise, the condition that ensures negative assortative matching for any distribution of types is stringent, requiring, for example, the absence of any complementarities for the case of urn-ball matching. Again, we show that for many search technologies (such as urn-ball) there exist particular distributions for which weaker requirements suffice.

\footnotetext{
${ }^{3}$ The models are not immediately comparable partly because random search requires a set based notion of assortative matching, while the frictionless benchmark and our model do not. Note also that the conditions in Shimer and Smith (2000) include log-supermodularity even of first and cross-partial derivatives, but not log-supermodularity. However, coupled with monotonicity as assumed throughout our model, log-supermodularity is implied by their conditions. We discuss the relation to Shimer and Smith (2000) and other work in Section 6.
} 
Our results hold for very general search technologies and match values. Yet, it turns out that a large class of widely used search technologies has a common condition, that of square-root-supermodularity. This is the case for any search technology that has bounds on its derivatives at zero and some curvature restriction, for example, the urn-ball search technology. In this class, the value of the elasticity at zero is always one-half. In contrast, the constant elasticity of substitution (CES) search technology satisfies the Inada conditions and, therefore, does not have bounded derivatives. Because its elasticity of substitution is constant, it separates the range of positive and negative sorting exactly.

Finally, we establish existence of a sorting equilibrium and show efficiency, that is, the planner's solution can be decentralized. While the efficiency properties of directed search models are well known (see, e.g., Moen (1997), Acemoglu and Shimer (1999b), and Shi (2001)), we discuss in particular the connection of our condition to the well known Hosios condition. Hosios' (1990) original contribution considers identical buyer and seller types, and relates the first derivative of the aggregate search technology to the match value. In our setting, this holds for each submarket. With heterogeneity, agents have a choice of which submarket to join. Our root-supermodularity condition ensures efficient sorting across submarkets by relating the elasticity of substitution of the aggregate search technology to the complementarities in the match value.

In the discussion section, we relate our model to existing results in the search literature. We discuss directed and random search, and the relationship of our model to the large literature on the foundations of competitive equilibria as limits of matching games with vanishing frictions. We consider a convergent sequence of search technologies in our static economy such that, in the limit, the short side of the market gets matched with certainty. To our knowledge, considering vanishing frictions as the limit of a sequence of static search technologies is new in this literature on foundations of competitive equilibrium. In the conclusions, we also highlight that our results do not only apply to search markets, but also shed some initial light on sorting in many-to-many matching markets.

\section{THE MODEL}

We cast our model in the context of a generic trading environment between buyers and sellers, as is often done in the directed search literature. This environment includes the labor market and many other markets with two-sided heterogeneity and search frictions. Our setup is chosen to be as general as possible and to encompass a broad class of different search technologies.

Players: There is a mass of heterogeneous sellers who are indexed by a type $y \in \mathcal{Y}$ that is observable. Let $S(y)$ denote the measure of sellers with types weakly below $y \in \mathcal{Y}$. We assume $\mathcal{Y}=[y, \bar{y}] \subset \mathbb{R}_{+}$and that $S(\bar{y})$ denotes the overall measure of sellers. Each seller has one good for sale. On the other 
side of the market there is a unit mass of buyers. Buyers differ in their valuation for the good, which is private information. Each buyer draws his type $x$ independently and identically distributed (i.i.d.) from distribution $B(x)$ on $\mathcal{X}=[\underline{x}, \bar{x}] \subset \mathbb{R}_{+} . S$ and $B$ are $C^{2}$, with strictly positive derivatives $s$ and $b$, respectively. It is convenient to think of a continuum of agents of each type, and of $b(x)$ and $s(y)$ as the size of the group of type $x$ buyers and type $y$ sellers.

Preferences: The value of a good consumed by buyer $x$ and bought from seller $y$ is given by $f(x, y)$, where $f$ is a strictly positive function $f: \mathbb{R}_{+}^{2} \rightarrow \mathbb{R}_{++}$. Conditional on consuming and paying a price $p$, the utility of the buyer is $f(x, y)-p$ and that of the seller is $p$. That is, agents have quasilinear utilities. We discuss broader preferences for the seller in the conclusion. We assume that $f$ is twice continuously differentiable in $(x, y)$. We consider indices $x$ and $y$ that are ordered such that they increase the utility of the buyer: $f_{x}>0, f_{y}>0$. The utility of an agent who does not consume is normalized to zero. Clearly, no trade takes place at prices below zero and above $f(\bar{x}, \bar{y})$, and we define the set of feasible prices as $\mathcal{P}=[0, f(\bar{x}, \bar{y})]$. All agents maximize expected utility.

Search Technology: The model is static. ${ }^{4}$ There are search frictions in the sense that with positive probability, a buyer does not get to match with the seller he has chosen. The extent of the frictions depends on the competition for the goods. We capture this idea of competition by considering the ratio of buyers to sellers, denoted by $\lambda \in[0, \infty]$, and refer to it as the expected queue length. This ratio varies in general with the quality of the good offered and the price posted. When a seller faces a ratio of $\lambda$, then he meets (and trades with) a buyer with probability $m(\lambda)$. The idea that relatively more buyers make it easier to sell is captured by assuming that $m:[0, \infty] \rightarrow[0,1]$ is a strictly increasing function. Analogously, buyers who want to trade at a price-quality combination that attracts a ratio $\lambda$ of buyers to sellers can buy with probability $q(\lambda)$, where $q:[0, \infty] \rightarrow[0,1]$ is a strictly decreasing function: when there are relatively more buyers, it becomes harder for them to trade. Trading in pairs requires that $q(\lambda)=m(\lambda) / \lambda$. We additionally impose the standard assumption that $m$ is twice continuously differentiable, strictly concave, and has a strictly decreasing elasticity.

Examples of Search Technologies: There are many ways to interpret and provide a microfoundation for the search technology. The most common one arises when buyers directly choose a seller but use an anonymous strategy in their selection. That means that once they decide on the quality-price combination, they choose one of the sellers with these characteristics at random. In a large market with many buyers and sellers, the probability that a seller has at least one buyer and can trade is approximately $m_{1}(\lambda)=1-e^{-\lambda}$. This search technology was first proposed by Butters (1977) (see also Peters (1991), Shi (2001), Shimer (2005)). Variations of this specification arise naturally, for

\footnotetext{
${ }^{4}$ We discuss our findings for steady states of a repeated model in the conclusion. See also our working paper version.
} 
example, when a fraction $1-\beta$ of the buyers gets lost on the way to the sellers, we have $m_{2}(\lambda)=1-e^{-\beta \lambda}$. Alternatively, if, at each price-quality combination, agents form pairs randomly, but trade only occurs when a seller is paired with a buyer (in the spirit of Kiyotaki and Wright (1993)), the matching probability is $m_{3}(\lambda)=\lambda /(1+\lambda)$.

Extensive Form and Trading Decisions: The extensive form of the market interaction has two stages. In stage 1, all sellers simultaneously post a price $p$ at which they are willing to sell the good. In stage 2, after observing the sellers' qualities and their posted prices $(y, p)$, buyers simultaneously decide where to attempt to buy, that is, each buyer chooses the quality-price combination $(y, p)$ that she seeks. A buyer for whom all the prices $p$ are too high can always choose the option of no trade, denoted by $\emptyset .^{5}$ A buyer who gets matched consumes the good and pays the posted price. Whether a buyer gets matched with a seller is determined by the search technology. This two-stage extensive form is in the spirit of, for example, Peters $(1991,2000)$ and Acemoglu and Shimer (1999a, 1999b). We denote by $G(y, p)$ and $H(x, y, p)$ the distribution of trading decisions of sellers and buyers, that is, $G(y, p)$ is the measure of sellers who offer a quality-price combination below $(y, p)$, and $H(x, y, p)$ is the measure of buyers with types below $x$ who attempt to buy a quality-price combination that is below $(y, p)$.

For many subsequent discussions, the marginals of these distributions are important; we denote them with subscripts. For example, $H_{\mathcal{X}}(x)$ is the fraction of buyers with type below $x$ and $H_{\mathcal{Y P}}(y, p)$ is the fraction of buyers that search for a quality below $y$ at a price below $p$. We impose the following two requirements. First, we require $G_{\mathcal{Y}}=S$ and $H_{\mathcal{X}}=B$, that is, the measure of traders coincides with the distribution in the population. Second, we require $H_{\mathcal{Y P}}$ to be absolutely continuous with respect to $G$, which means that if there are no sellers who have chosen prices in some set, then no buyers will try to buy from that set. This will enable us to use the Radon-Nikodym derivative below.

Equilibrium: Our equilibrium concept follows the literature on large games (see, e.g., Mas-Colell (1984)), where the payoff of each individual is determined only by his own decision and by the distribution of trading decisions $G$ and $H$ in the economy, which in turn have to arise from the optimal decisions of the individual traders. ${ }^{6}$ To define the expected payoffs for each agent given $G$ and $H$, let the function $\Lambda_{G H}: \mathcal{Y} \times \mathcal{P} \rightarrow[0, \infty]$ denote the expected queue length at each quality-price combination. Along the support of the sellers' trading distribution $G$ it is given by the Radon-Nikodym derivative

\footnotetext{
${ }^{5}$ To make the choice of no trade consistent with the rest of our notation, let $\emptyset=\left(\emptyset_{y}, \emptyset_{p}\right)$, where $\emptyset_{y}<y$ denotes a nonexistent quality and $\emptyset_{p}<0$ denotes a nonexistent price, and the trading probability at $\varnothing$ is zero.

${ }^{6} \mathrm{We}$ are grateful to Michael Peters for pointing out to us this approach, which brings the competitive search model in line with the standard game theoretic approach to large markets.
} 
$\Lambda_{G H}=d H_{\mathcal{Y P}} / d G .^{7}$ Along the support of $G$, we can define the expected payoff of sellers as

$$
\pi(y, p, G, H)=m\left(\Lambda_{G H}(y, p)\right) p
$$

and the expected pay off of buyers as

$$
u(x, y, p, G, H)=q\left(\Lambda_{G H}(y, p)\right)[f(x, y)-p] .
$$

So far the payoffs are only determined on the path of play, since the buyerseller ratio $\Lambda_{G H}$ is only well defined there. We extend the payoff functions by extending the queue length function $\Lambda_{G H}$ to all of $\mathcal{Y} \times \mathcal{P}$. A seller who contemplates a deviation and offers a price different from all other sellers, that is, $(y, p) \notin \operatorname{supp} G$, has to form a belief about the queue length that he will attract. We follow the literature (e.g., McAfee (1993), Acemoglu and Shimer (1999b), Shimer (2005)) by imposing restrictions on beliefs in the spirit of subgame perfection: the seller expects a queue length $\Lambda_{G H}(y, p)$ larger than zero only if there is a buyer type $x \in \mathcal{X}$ who is willing to trade with him. Moreover, he expects the highest queue length for which he can find such a buyer type, which means that he expects buyers to queue up for the job until it is no longer profitable for them to do so. Formally, that means that

$$
\begin{aligned}
\Lambda_{G H}(y, p)= & \sup \left\{\lambda \in \mathbb{R}_{+}:\right. \\
& \left.\exists x ; q(\lambda)[f(x, y)-P] \geq \max _{\left(y^{\prime}, p^{\prime}\right) \in \operatorname{supp} G} u\left(x, y^{\prime}, P^{\prime}, G, H\right)\right\}
\end{aligned}
$$

if that set is nonempty and $\Lambda_{G H}(y, p)=0$ otherwise. This extension defines the queue length and thus the matching frictions and payoffs on the entire domain. ${ }^{8}$ Here the queue length function $\Lambda_{G H}$ acts similar to Rosen's (1974) hedonic price schedule in the sense that individuals take this function as given, and an equilibrium simply states that all trading decisions according to $G$ and $H$ are indeed optimal given the implied queue lengths.

DEFINITION 1: An equilibrium is a pair of trading distributions $(G, H)$ such that the following conditions hold:

(i) Seller Optimality: $(y, p) \in \operatorname{supp} G$ only if $p$ maximizes (1) for $y$.

${ }^{7}$ On the support of $G$, the Radon-Nikodym derivative is well defined, up to a zero measure set: any two derivatives coincide almost everywhere. To achieve everywhere well defined payoffs in (1) and (2), assume some rule that selects a unique $\Lambda_{G H}$ on $\operatorname{supp} G$ for each $(G, H)$. For our existence proof, we require the selection to be continuous and differentiable wherever possible on $\operatorname{supp} G$, as this will select the derivative that we construct.

${ }^{8}$ For particular microfoundations of the matching function in an economy with one-sided heterogeneity, Peters $(1991,1997 \mathrm{a}, 2000)$ showed that the specification of the matching frictions in (3) indeed arises the equilibrium path after a deviation by an individual seller. 
(ii) Buyer Optimality: ( $x, y, p) \in \operatorname{supp} H$ only if $(y, p)$ maximizes (2) for $x$.

Assortative Matching: Our main focus is on the sorting of buyers across sellers. In ex ante terms, an allocation is not one-to-one since the ratio of buyers to sellers is, in general, different from 1 . Therefore, we define sorting in terms of the distribution of visiting decisions of buyers $H$. Consider active buyer types $x$ who choose to be in the market rather than taking their outside option $((x, \emptyset) \notin \operatorname{supp} H)$. We say that $H$ entails assortative matching if there exists a strictly monotone function $\nu$ that maps these buyer types into $\mathcal{Y}$ such that $H_{\mathcal{X Y}}(x, \nu(x))=B(x)$ for all active buyer types. This means that $\nu(x)$ is the seller type with which buyer type $x$ would like to trade. We say that matching is positive assortative if $\nu$ is strictly increasing and is negative assortative if it is strictly decreasing. Since $\nu$ is strictly monotone, it is uniquely characterized by its inverse $\mu \equiv \nu^{-1}$, where $\mu(y)$ denotes the buyer type that visits seller $y$. Throughout we will consider this inverse and call it the assignment.

\section{THE MAIN RESULTS}

In equilibrium, an individual seller of type $y$ takes the trading distributions $G$ and $H$ as given, and according to part (i) of the equilibrium definition, his pricing decision solves $\max _{p} m\left(\Lambda_{G H}(y, p)\right) p$. This seller can set a price that does not attract any buyers $\left(\Lambda_{G H}(y, p)=0\right)$ or he can set a price that attracts buyers $\left(\Lambda_{G H}(y, p)>0\right)$ and we can substitute $(3)$, which holds by assumption outside the support of $G$ and also by equilibrium condition (ii) on the support of $G$. Therefore, the seller's problem can be written as

$$
\max _{\lambda, p}\left\{m(\lambda) p: \lambda=\sup \left\{\lambda^{\prime}: \exists x ; q\left(\lambda^{\prime}\right)[f(x, y)-p] \geq U(x, G, H)\right\}\right\},
$$

where we introduced $U(x, G, H) \equiv \max _{\left(y^{\prime}, p^{\prime}\right) \in \operatorname{supp} G} u\left(x, y^{\prime}, p^{\prime}, G, H\right)$ to denote the highest utility that a buyer of type $x$ can obtain. By equilibrium condition (ii), $U(x, G, H)$ is continuous. Therefore, for sellers who trade with positive probability, this problem is equivalent to

$$
\max _{x, \lambda, p}\{m(\lambda) p: q(\lambda)[f(x, y)-p]=U(x, G, H)\} .
$$

This maximization problem has a natural interpretation that is common to much of the literature on competing mechanism design. It states that a seller can choose prices and trading probabilities as well as the buyer type that he wants to attract, as long as the utility for this buyer is as large as the utility that he can get by trading with other sellers. Note also that $(x, y, p)$ cannot be in the support of the buyers' equilibrium trading strategy $H$ if there does not exist a $\lambda$ such that $(x, \lambda, p)$ solves (4) for $y$, since the price and associated queue length offered by $y$ will not allow buyer $x$ to obtain his expected equilibrium utility $U(x, G, H)$. To simplify notation in what follows, we suppress the dependence of the variables on $G$ and $H$ when there is no danger of confusion. 
We will now derive a necessary condition for assortative matching. For expositional purposes, we focus on a particular class of equilibria in this derivation that fulfill a number of differentiability conditions. Consider a candidate equilibrium $(G, H)$ that is assortative, that is, it permits a strictly monotone assignment $\mu(y)$, and has a unique price $p(y)$ offered by seller type $y$, with both $\mu(y)$ and $p(y)$ differentiable. ${ }^{9}$ The focus on a differentiable equilibrium is just for convenience of exposition in the main body. The formal proofs do not assume differentiability a priori.

For any seller $y$ who trades at an interior queue length, we can use the constraint to substitute out the price in (4). Since $m(\lambda)=\lambda q(\lambda)$, this yields

$$
\max _{x, \lambda} m(\lambda) f(x, y)-\lambda U(x) .
$$

Along the equilibrium path, seller $y$ 's assigned buyer type $\mu$ (i.e., $\mu(y))$ and his queue length $\Lambda$ (i.e., $\Lambda(y, p(y)))$ solve this program and are characterized by its first-order conditions

$$
\begin{aligned}
& m^{\prime}(\Lambda) f(\mu, y)-U(\mu)=0, \\
& m(\Lambda) f_{x}(\mu, y)-\Lambda U^{\prime}(\mu)=0 .
\end{aligned}
$$

The first-order conditions only characterize an optimal choice if the secondorder condition is satisfied. To verify the second-order condition, we derive the Hessian along the equilibrium path:

$$
\left(\begin{array}{cc}
m^{\prime \prime}(\Lambda) f(\mu, y) & m^{\prime}(\Lambda) f_{x}(\mu, y)-U^{\prime}(\mu) \\
m^{\prime}(\Lambda) f_{x}(\mu, y)-U^{\prime}(\mu) & m(\Lambda) f_{x x}(\mu, y)-\Lambda U^{\prime \prime}(\mu)
\end{array}\right) .
$$

The term $m^{\prime \prime}(\Lambda) f(\mu, y)$ is strictly negative and the point $(\Lambda, \mu)$ is a local maximum only if the determinant of the Hessian is positive:

$$
\begin{gathered}
m^{\prime \prime}(\Lambda) f(\mu, y)\left(m(\Lambda) f_{x x}(\mu, y)-\Lambda U^{\prime \prime}(\mu)\right) \\
-\left(m^{\prime}(\Lambda)-m(\Lambda) / \Lambda\right)^{2} f_{x}(\mu, y)^{2} \geq 0,
\end{gathered}
$$

where in the last term of this inequality we have substituted $U^{\prime}$ from (7). Totally differentiating (7) with respect to $y$ and using (7) yields the expression

$$
\begin{aligned}
U^{\prime \prime}(\mu)= & \frac{m(\Lambda)}{\Lambda} f_{x x}(\mu, y) \\
& +\frac{1}{\Lambda \mu^{\prime}}\left(\left(m^{\prime}(\Lambda)-\frac{m(\Lambda)}{\Lambda}\right) f_{x}(\mu, y) \frac{d \Lambda}{d y}+m(\Lambda) f_{x y}(\mu, y)\right) .
\end{aligned}
$$

\footnotetext{
${ }^{9}$ We require this only for those types that trade with strictly positive probability. A unique price $p(y)$ means that $(y, p(y)) \in \operatorname{supp} G$ and $\left(y, p^{\prime}\right) \notin \operatorname{supp} G$ for any other $p^{\prime} \neq p(y)$. Finally, we note that $\mu(y)$ and $p(y)$ are differentiable only if $U(x, G, H)$ is twice differentiable in $x$ and $\Lambda(y, p(y))$ is totally differentiable in $y$, as shown in (10) and (11) below.
} 
Totally differentiating (6) with respect to $y$ and substituting (7) yields an expression for the change of the queue length along the equilibrium path:

$$
\begin{aligned}
\frac{d \Lambda}{d y}= & -\frac{1}{m^{\prime \prime}(\Lambda) f(\mu, y)} \\
& \times\left[\left(m^{\prime}(\Lambda)-\frac{m(\Lambda)}{\Lambda}\right) f_{x}(\mu, y) \mu^{\prime}+m^{\prime}(\Lambda) f_{y}(\mu, y)\right] .
\end{aligned}
$$

Substituting (10) and (11) into (9) allows us to cancel terms, and after rearranging and multiplying by $\mu^{\prime}(y)^{2}$, we are left with

$$
\mu^{\prime}(y)\left[f_{x y}(\mu, y)-\frac{m^{\prime}(\Lambda)\left(\Lambda m^{\prime}(\Lambda)-m(\Lambda)\right)}{\Lambda m^{\prime \prime}(\Lambda) m(\Lambda)} \frac{f_{x}(\mu, y) f_{y}(\mu, y)}{f(\mu, y)}\right] \geq 0 .
$$

To satisfy the second-order condition, both terms in (12) must have identical signs. Under PAM $\left(\mu^{\prime}>0\right)$, the term in square brackets has to be positive; under NAM $\left(\mu^{\prime}<0\right)$, it has to be negative. Defining

$$
a(\lambda) \equiv \frac{m^{\prime}(\lambda)\left(m^{\prime}(\lambda) \lambda-m(\lambda)\right)}{\lambda m(\lambda) m^{\prime \prime}(\lambda)},
$$

the following lemma follows immediately.

LEMMA 1: In any differentiable equilibrium that satisfies positive assortative matching,

$$
\frac{f_{x y}(\mu, y) f(\mu, y)}{f_{y}(\mu, y) f_{x}(\mu, y)} \geq a(\Lambda)
$$

has to hold along the equilibrium path, with the opposite sign in any differentiable equilibrium with negative assortative matching.

This condition is stronger than standard supermodularity, because our assumptions on the search technology imply that $a(\lambda) \in[0,1]$ for all $\lambda .^{10} \mathrm{~A}$ related but different condition was reported by Shi (2001) for a specific directed search model. His condition arises as a special case of (14), as we discuss in more detail in Section 6. The benefit of expression (14) is that it provides a

\footnotetext{
${ }^{10}$ One can rewrite (13) as $a(\lambda)=m^{\prime}(\lambda) q^{\prime}(\lambda) /\left(m^{\prime \prime}(\lambda) q(\lambda)\right)$, and our assumptions on the search technology immediately yield $a(\lambda)>0$ for all $\lambda \in(0, \infty)$. Furthermore, some straightforward algebra shows that a strictly decreasing elasticity of $m$ implies that $a(\lambda)<1$ for all $\lambda \in(0, \infty)$. More details are presented in the published working paper version. All results in this paper obtain even without the standard assumption that the elasticity of $m$ is decreasing, only that the right-hand side of condition (14) might be larger than 1 , which requires stronger supermodularity conditions.
} 
clear economic interpretation of the trade-offs for sorting in markets in which both search frictions and complementarities in values are present.

The economic insight of Lemma 1 becomes transparent when we interpret condition (14) in terms of the aggregate search technology $M$. This aggregate search technology is defined as the total number of matches that arise when $\beta$ buyers are in a market with $\sigma$ sellers, that is, $M(\beta, \sigma)=\sigma m(\beta / \sigma)$. Substituting for $M$ in (14) delivers the condition

$$
\frac{f_{x y}(\mu, y) f(\mu, y)}{f_{x}(\mu, y) f_{y}(\mu, y)} \geq \frac{M_{b}(\Lambda, 1) M_{s}(\Lambda, 1)}{M_{b s}(\Lambda, 1) M(\Lambda, 1)} .
$$

The right-hand side measures the elasticity of substitution of the aggregate search technology $M$ denoted by $\mathrm{ES}_{M} \cdot{ }^{11}$ When $f$ is constant returns, the lefthand side measures the inverse of the elasticity of substitution of the match value function $f$ denoted by $\mathrm{ES}_{f}$ (see Hicks (1932)). The condition highlights the nature of the trade-off between match value and trading security. To obtain PAM, the inverse of the elasticity of substitution of the surplus function $\mathrm{ES}_{f}$ must exceed the elasticity of substitution of the search technology $\mathrm{ES}_{M}: \mathrm{ES}_{f}^{-1} \geq \mathrm{ES}_{M}$.

If different markets are very substitutable (high $\mathrm{ES}_{M}$ ), then $x$ and $y$ have to be strong complements (high $f_{x y}$ and, therefore, low $\mathrm{ES}_{f}$ ). The latter corresponds to the gain in match value due to complementarity and reflects the marginal increase in output from increasing both types. That degree of complementarity must offset the gains from using additional low types to help high types trade. If the elasticity of substitution $\mathrm{ES}_{M}$ is large, additional low types are very efficient in providing such trading security. Therefore, complementarities in production have to be strong to nevertheless induce PAM. For aggregate search technologies with a constant elasticity of substitution, the righthand side of (14) is constant and determines the degree of supermodularity required of $f$. In general, the supremum and infimum of that elasticity become of importance. Let $\bar{a}=\sup a(\lambda) ; \underline{a}=\inf a(\lambda)$. Both lie in [0,1]. We discuss some specific search technologies in depth in the next section, after presenting the main results on sorting.

To state our main result, we first introduce a notion of the degree of supermodularity. Clearly, for condition (14) to hold, it does not suffice that function $f$ is simply supermodular. For any two buyer and seller types $x_{2}>x_{1}$ and $y_{2}>y_{1}$, supermodularity means that the total value when the high types trade and when the low types trade is higher than when there is cross-trade (low with high and vice versa): $f\left(x_{2}, y_{2}\right)+f\left(x_{1}, y_{1}\right) \geq f\left(x_{2}, y_{1}\right)+f\left(x_{1}, y_{2}\right)$. This also means that the extreme values (very high $f$ and very low $f$ ) on the left-hand side of the inequality are jointly higher than the intermediate values on the

\footnotetext{
${ }^{11}$ We are grateful to John Kennan for pointing out that $a(\lambda)$ is equal to the elasticity of substitution of the aggregate search technology $\mathrm{ES}_{M}$.
} 
right. The equivalent condition when $f(x, y)$ is differentiable is that the crosspartial is positive: $f_{x y}(x, y)>0$. Such a condition only includes the gains if agents trade, but in our setting we also need to consider the losses if agents do not trade. These losses especially affect the high types and gives them extra incentives to ensure trade by attracting (many) low types. We therefore need a stronger condition for positive sorting, and the idea that assortative matching becomes harder can be captured by strengthening the supermodularity condition as follows. Let $g$ be a concave function and require that $g \circ f$ be supermodular, that is, $g \circ f\left(x_{2}, y_{2}\right)+g \circ f\left(x_{1}, y_{1}\right) \geq g \circ f\left(x_{2}, y_{1}\right)+g \circ f\left(x_{1}, y_{2}\right)$. Concavity affects extreme values on the left of the inequality more than intermediate values on the right, which makes this condition of assortative matching more difficult to fulfill. This is easiest to see in the differential version of this inequality: $\partial^{2} g(f(x, y)) / \partial x \partial y \geq 0$ or, equivalently,

$$
\frac{f_{x y}(x, y) f(x, y)}{f_{x}(x, y) f_{y}(x, y)} \geq-\frac{g^{\prime \prime}(f(x, y)) f(x, y)}{g^{\prime}(f(x, y))} .
$$

Exactly how much more difficult it is to sustain this inequality is captured by the (relative) Arrow-Pratt measure of the transform $g$ on the right-hand side of (16). For example, this measure is 0 if $g$ is a linear transformation and it is 1 if $g$ is a log-transformation. Compare this inequality with (14). By virtue of the sup (or inf) of $a$, the right-hand side of (14) is a constant in the unit interval. A constant right-hand side of (16) with similar magnitude is exactly induced by the transformation $g(f)=\sqrt[n]{f}$. We say that function $f$ is $n$-root-supermodular with coefficient $n \in(1, \infty)$ if $\sqrt[n]{f}$ is supermodular. By (16), this requires that the cross-partial derivative of $f$ is sufficiently large, that is,

$$
\frac{f_{x y}(x, y) f(x, y)}{f_{x}(x, y) f_{y}(x, y)} \geq 1-n^{-1} .
$$

This captures standard supermodularity when $n=1$ and approaches logsupermodularity as $n \rightarrow \infty$. We can now state the main result:

THEOREM 1: For any type distributions $B$ and $S$ any equilibrium is positive assorted if and only if function $f$ is n-root-supermodular, where $n=(1-\bar{a})^{-1}$. For any type distributions $B$ and $S$ any equilibrium is negative assorted if and only if function $f$ is nowhere n-root-supermodular, where $n=(1-\underline{a})^{-1}$.

See the Appendix for the proof.

The proof focusses on positive assortative matching and consists of two parts. First, we show that (strict) $n$-root-supermodularity implies positive assortative matching. Since we want to rule out other equilibria that might be nonassortative, we cannot work with a monotone differentiable assignment $\mu$; therefore, we deploy a different proof technique than in the derivation of condition 
(14). Second, we show that positive assortative matching for all type distributions implies that $f$ has to be (weakly) $n$-root-supermodular. Here the proof works by contradiction: If $f$ is not $n$-root-supermodular at some point $(x, y)$ in the domain, then we can construct a type distribution such that types in the neighborhood of $(x, y)$ trade at a queue length $\lambda$ with $a(\lambda)$ close enough to $\bar{a}$ and, therefore, larger than the degree of root-supermodularity of $f$. This directly contradicts the condition for PAM in Lemma 1 for differentiable equilibria, and a similar contradiction can be derived for nondifferential equilibria. Key here is that the result holds for all distributions. For a particular type distribution, PAM may arise with less complementarities, because the value of $\bar{a}$ might not be attained in equilibrium. The proofs in the case of negative assortative matching are completely analogous and are omitted for brevity.

The theorem establishes a dividing range between positive and negative sorting. This dividing range collapses to a line when $\underline{a}=\bar{a}$ (see also Section 4 where we discuss constant elasticity of substitution matching technologies). Such a sharp cutoff is also a feature of Becker's (1973) frictionless theory, but our cutoff is shifted toward larger complementarities. In our environment, the fact that low types are valuable because they can help facilitate trade for the high types has the novel implication that under $\underline{a}>0$, for all type distributions, NAM obtains even if $f$ is strictly supermodular as long as it is nowhere $n$-rootsupermodular $\left(n=(1-\underline{a})^{-1}\right)$. On the other hand, if $\underline{a}<\bar{a}$, then the areas of positive and negative sorting are not as sharply divided. This is the case specifically for those search technologies such as urn-ball technology that have $\underline{a}=0$. Still, any $f$ that is weakly submodular $\left(f_{x y} \leq 0\right)$ induces NAM. ${ }^{12}$

The conditions in Theorem 1 are particularly strong so as to ensure sorting under any possible type distribution. This gives us useful bounds, but these bounds might not be necessary for given type distributions. If the elasticity of substitution is not constant, it may be the case that neither the supremum $\bar{a}$ nor the infimum $\underline{a}$ are reached on the equilibrium path. This explains the weaker notion in an example by Shi (2001), who considered the urn-ball search technology and a given seller type distribution. His Example 5.2 has negative sorting despite $f_{x y}>0$ and $\underline{a}=0$. We formalize this in the next proposition.

PROPOSITION 1: Consider a search technology such that a $(\cdot)$ is not constant:

(i) There exist distributions $B$ and $S$ and functions $f$ that are nowhere n-rootsupermodular $\left(n=(1-\bar{a})^{-1}\right)$ such that any equilibrium exhibits positive assortative matching.

\footnotetext{
${ }^{12}$ In general, negative assortative matching has to arise under the strict inequality $f_{x y}<$ $\underline{a} f_{x} f_{x} f^{-1}$. The case of $\underline{a}=0$ is special because negative assortative matching is ensured even when $f_{x y}=0$, since in this case our assumptions on the search technology still imply $a(\lambda)>0$ whenever $\lambda \in(0, \infty)$. Therefore, for all types that trade with positive probability $(\lambda \neq 0, \infty)$, the elasticity is strictly positive and the proof technique immediately extends to this case.
} 
(ii) There exist distributions $B$ and $S$ and strictly n-root-supermodular $(n=$ $\left.(1-\underline{a})^{-1}\right)$ functions $f$ such that any equilibrium exhibits negative assortative matching.

See the Appendix for all proposition proofs.

Finally, we establish existence of a (differentiable) equilibrium. Existence in our setup is more complicated than in frictionless matching models because we cannot employ the standard measure-consistency condition. In our setup, it is possible that more agents from one side attempt to trade with the other, and this imbalance is absorbed through different trading probabilities. ${ }^{13}$ The system retains tractability when we impose the sufficient conditions for assortative matching (either PAM or NAM), in which case we can exploit differential equation (11) to construct the equilibrium path along the first-order condition and use the sufficient conditions to show that deviations are not profitable.

PROPOSITION 2: If the function $f$ satisfies n-root-supermodularity for $n=(1-$ $\bar{a})^{-1}$ (or nowhere n-root-supermodularity for $\left.n=(1-\underline{a})^{-1}\right)$, then for any type distributions $B$ and $S$, there exists a differentiable equilibrium.

\section{CHARACTERIZATION}

In this section we discuss the characterization of the equilibrium. We consider two particular classes of commonly used search technologies that allow particularly sharp bounds on the degree of supermodularity: those that are bounded and imply square-root-supermodularity, and those that have a constant elasticity of substitution. We then investigate the properties of the equilibrium price schedule.

\subsection{Common Search Technologies}

Square-root-supermodularity is the property that applies to a large class of search technologies, including those that are built on microfoundations, such as the example search technologies $m_{1}, m_{2}$, and $m_{3}$ outlined above. The class is characterized by technologies with local bounds on the derivatives and enough

\footnotetext{
${ }^{13}$ In frictionless one-to-one matching models with a continuum of agents, existence can be proven by considering the efficient allocation, which can be characterized by a linear program for which existence is proven by Kantorovich (1958). The efficient allocation in our setting resembles Kantorovich's optimal transportation problem, with the one major difference that it is not a linear program since the buyer-seller ratio enters the objective (see (18)). Interpretation of a submarket as a coalition of many buyers and sellers in the spirit of the many-to-many matching literature still does not allow us to adopt existence proofs from this literature, since the proofs we are aware of rely on finite coalitions of bounded size, whereas in our setting submarkets with uncountably many buyer and seller arise.
} 
curvature. To lay this out formally, it will be convenient to consider the matching probability $q(\lambda)$ of the buyers, which is linked to the matching probability of the sellers via $m(\lambda)=\lambda q(\lambda)$.

PROPOSITION 3-Square-Root-Supermodularity: Let $\left|q^{\prime}(0)\right|>0$ and $\left|q^{\prime \prime}(0)\right|<\infty$, and let $1 / q$ be convex. For any type distributions $B$ and $S$ any equilibrium exhibits $P A M$ if and only if $f(x, y)$ is square-root-supermodular.

Understanding what drives the sorting pattern is motivated by the relation between the complementarities in match value and the elasticity of substitution of the search technology. It is then somewhat striking that in such a large class of search technologies - arguably the most relevant ones-all depend exactly on that same condition: square-root supermodularity. The explanation for this is entirely driven by the value of the elasticity of substitution at zero. The bounds on the derivatives imply that it is necessarily pinned down at one half, which turns out to be a general property of homothetic functions as can be seen in the proof. This makes square-root-supermodularity necessary. The curvature restriction is equivalent to the requirement that the elasticity of substitution does not exceed one-half at some point other than zero and, therefore, square-root-supermodularity is sufficient.

Constant elasticity of substitution (CES) matching technologies are often assumed for their simplicity. Since the elasticity of substitution is invariant, they can be represented by $m(\lambda)=\left(1+k \lambda^{-r}\right)^{-1 / r}$, where $r>0$ and $k>1$. The associated aggregate CES search technology for a given number of buyers and sellers $\beta$ and $\sigma$ is defined as (see, among others, Menzio (2007)):

$$
M(\beta, \sigma)=\left(\beta^{r}+k \sigma^{r}\right)^{-1 / r} \beta \sigma .
$$

The elasticity of substitution is given by $\mathrm{ES}_{M}=(1+r)^{-1}$. The CES matching technologies do not fall into the previous category because either the bounds at zero are violated or the curvature restriction does not hold. The exception is the knife-edge case with $r=1$ that corresponds to (a variation of) the matching technology $m_{3}=\lambda /(\lambda+k)$ that is CES.

The CES search technology nonetheless gives very sharp predictions on the necessary and sufficient conditions for positive and negative assortative matching: PAM arises when $f(x, y)$ is $n$-root-supermodular and NAM arises when $f(x, y)$ is nowhere $n$-root-supermodular, where $n=(1+r) / r$ is the same in both cases. It is important to stress here that $n$-root-supermodularity is a necessary condition for positive assortative matching even if we consider only a particular type distribution. This is stronger than our Theorem 1, and arises exactly because the elasticity is constant and we do not have to worry whether the supremum is actually realized on the path of play. Moreover, since Theorem 1 ensures NAM for any given distribution, it also provides direct evidence that NAM will arise for any type distributions even if the match value function 
is (moderately) supermodular, since the elasticity of substitution is bounded away from zero. The class of CES search technologies spans the entire range of $n$-root-supermodularity, from supermodularity to log-supermodularity, as stated in the next corollary to Theorem 1.

COROLLARY 1: Let the search technology be CES with elasticity $\mathrm{ES}_{M}$. Then a necessary and sufficient condition for PAM is one of the following cases:

(i) Supermodularity if $\mathrm{ES}_{M} \simeq 0$ (Leontief).

(ii) Square-root-supermodularity if $\mathrm{ES}_{M}=\frac{1}{2}\left(m_{3}\right)$.

(iii) Log-supermodularity if $\mathrm{ES}_{M} \simeq 1$ (Cobb-Douglas).

\subsection{The Equilibrium Price Schedule}

Our results are cast in terms of the monotonicity of the allocation, offering sharp predictions on assortative matching. In contrast, equilibrium does not provide equally general predictions in terms of the monotonicity of the price schedule. Equilibrium prices can be both increasing and decreasing in type, because agents are compensated through both prices and trading probabilities. This is not the case in the frictionless model of Becker (1973). There, $p^{\prime}(y)=$ $f_{y}>0$, that is, the slope of the price schedule is equal to the marginal product of being matched with a better seller. For our setting, we derive the equilibrium price schedule in the Appendix. It satisfies

$$
p^{\prime}(y)=f_{y}+a\left[\left(1-\eta_{m}\right) f_{x} \mu^{\prime}-\eta_{m} f_{y}\right],
$$

where $\eta_{m}=\lambda m^{\prime} / m$ is the elasticity of $m, a$ is the elasticity of substitution, and $\mu^{\prime}$ is the change of trading partner along a differentiable equilibrium. This price schedule decentralizes the efficient allocation (Proposition 4 below). It reflects the marginal benefit conditional on matching, but additionally reflects the marginal benefit from the change in the probability of a match. In this world with trading frictions, sellers can be rewarded through higher prices or better trading probabilities. Higher seller types obviously have to make higher equilibrium profits, yet this increase may be due more to the second source than to the first and equilibrium prices can actually be declining. For this to happen, the trading probabilities have to rise substantially, though, which is only possible under negative assortative matching.

Inspection of equation (17) immediately reveals that under PAM (with $\mu^{\prime}>0$ ), the price schedule is increasing in firm type. The effect introduced by the search frictions can never be so strong that prices actually decrease: both $a$ and $\eta_{m}$ are in $[0,1]$, and as a result the aggregate sign on the $f_{y}$ term as determined by $\left(1-a \eta_{m}\right)$ is positive. This is not necessarily true under NAM, where $\mu^{\prime}<0$. Prices can then be decreasing, for example, consider some fixed type distributions and $f_{y}$ sufficiently small. Then sellers must make nearly identical 
profits. If buyer types remain important $\left(f_{x} \gg 0\right)$, high buyer types obtain substantially higher equilibrium utility than low buyer types. Therefore, in equilibrium, low seller types leave high utility to their (high-type) customers and obtain low queue length since $d \Lambda / d y$ in equation (11) is positive under NAM. To make nearly equal profits according to (4), the low seller types have to charge a higher price in equilibrium. Since the price change (17) does not depend directly on the cross-partial, particularly simple examples of this phenomenon can be constructed with modular match values $\left(f_{x y}=0\right)$.

Finally, it is instructive to consider the price function in a symmetric world. Suppose there is symmetry between buyers and sellers in the match value function $f(x, y)$ and in the aggregate search technology $M(\beta, \sigma)$, and the type distributions are identical for buyers and sellers. Then it is straightforward to show that under root-supermodularity and, therefore, PAM, a "symmetric" equilibrium exists with $\mu(y)=x$ and a constant queue length $\lambda=1$ along the equilibrium path. Since symmetry of $M$ implies that $\eta_{m}=1 / 2$, the pricing function reduces exactly to the marginal value of Becker (1973), that is, $p^{\prime}=f_{y}$. This highlights the fact that the effect on prices due to search frictions is only prevalent in the presence of asymmetries. In a positively assorted equilibrium, under symmetry, the effects of frictions exactly cancel out.

\section{EFFICIENCY OF THE DECENTRALIZED ALLOCATION}

Consider a planner who chooses trading distributions $(G, H)$ to maximize the surplus in the economy, subject to the same search technology. The planner maximizes

$$
\begin{aligned}
\max _{G, H} \int q\left(\Lambda_{G H}(y, p)\right) f(x, y) d H \\
\text { s.t. } \quad G_{\mathcal{Y}}=S, H_{\mathcal{X}}=B, \Lambda_{G H}=d H_{\mathcal{Y P}} / d G,
\end{aligned}
$$

where the constraints correspond to the restrictions in the decentralized economy. Prices simply constitute transfers between agents and, therefore, they do not enter the planner's objective directly. They do allow the planner to let identical sellers trade at different queue lengths $\Lambda(y, p)$ and $\Lambda\left(y, p^{\prime}\right)$ with potentially different buyers, which is also possible in the decentralized economy. Since in the planner's problem prices play no direct role, we could as well have indexed the queue length by some other label such as a "location" instead of prices.

PROPOSITION 4: If $f$ is strictly $n$-root-supermodular with $n=(1-\bar{a})^{-1}$ (nowhere $n$-root-supermodular with $n=(1-\underline{a})^{-1}$ ), then any solution to the planner's problem is positive (negative) assorted and can be decentralized as an equilibrium. 
This result is in line with the efficiency properties of directed search models in general; see for example, Moen (1997), Acemoglu and Shimer (1999b), and Shi (2001). It is worth highlighting this efficiency property, because it allows us to interpret our sorting condition from an efficiency point of view.

Our result provides a condition that augments the standard Hosios (1990) condition for efficiency by relating different submarkets. The Hosios (1990) condition holds for a particular $(x, y)$ market and equates the social contribution to match formation with the split of the surplus between buyer and seller. In our decentralized equilibrium, substituting (6) into (5) yields the Hosios condition, which can be rewritten to say that seller $y$ 's equilibrium profits are $M_{s}(\Lambda, 1) f(x, y)$ and reflect his marginal contribution to match creation. With two-sided heterogeneity, the issue of efficiency hinges on which $(x, y)$ combinations trade in equilibrium. Our contribution is to show that this is governed not by the derivative of the aggregate matching technology $M$, but by its elasticity of substitution $a(\lambda)$.

The Hosios condition is usually associated with the elasticity $\eta_{m}$ of the individual search technology $m$ since $M_{s}=1-\eta_{m}$. A similar connection exists in our setting between the elasticity of substitution of $M$, denoted by $a$, and the elasticity $\eta_{m}$ of the individual matching technology $m$. To see this, observe that

$$
a(\lambda) \equiv \frac{m^{\prime}(\lambda)\left(m^{\prime}(\lambda) \lambda-m(\lambda)\right)}{\lambda m(\lambda) m^{\prime \prime}(\lambda)}=\frac{1-\eta_{m}(\lambda)}{\eta_{m^{\prime}}(\lambda)} .
$$

The first equality is the condition we derived above in equation (13). The second equality follows immediately after rearranging terms, where $\eta$ denotes the elasticity of the subscripted function: $\eta_{m}=\frac{\lambda m^{\prime}}{m}$ and $\eta_{m^{\prime}}=\frac{\lambda m^{\prime \prime}}{m^{\prime}}$. As with the Hosios condition, the condition here depends on the elasticity via $1-\eta_{m}$, which captures the marginal effect on the search technology. In addition, it depends on $\eta_{m^{\prime}}$, which captures the second degree marginal effect on the search technology. This effect governs how the matching probability changes as we move across different matched pairs. The latter effect is obviously absent with homogeneous types and, therefore, in the standard Hosios condition.

\section{DISCUSSION OF RELATED LITERATURE}

We relate our findings to models and results from three distinct literatures.

\subsection{Directed Search}

There is an extensive literature on directed search with and without twosided heterogeneity. Contributions range from work that provides a rationale for unemployment in the labor market and waiting times in the product market (for example, Peters (1991, 1997b, 2000, 2007), Acemoglu and Shimer (1999a, 1999b), Burdett, Shi, and Wright (2001), Shi (2001), Mortensen and Wright 
(2002), Galenianos and Kircher (2009), Kircher (2009), and Delacroix and Shi (2006)) to work that models more elaborate trading mechanisms (such as McAfee (1993), Peters (1997a), Shi (2002), Shimer (2005), and Eeckhout and Kircher (2010)).

Here we focus our attention on specific aspects of the most closely related paper by Shi (2001). Shi was the first researcher to show that, in an environment with directed search, supermodularity is not sufficient to attain PAM. He assumed that firms can freely enter with type $y$ if they pay some entry cost $C(y)$. He derived a condition that requires $f_{x y}$ to be sufficiently large that is seemingly different from ours. Here we show that our findings are consistent. His condition is

$$
\frac{f f_{x y}}{f_{x} f_{y}}>\frac{C f_{y}\left(f_{y}-C_{y}\right)}{C_{y}\left(f C_{y}-C f_{y}\right)} .
$$

The strength of this condition (i.e., the magnitude of the right-hand side) cannot readily be evaluated. Moreover, this condition seems not to depend on the search technology $m$, which is in apparent contradiction with our results. Our results imply that sorting depends on the elasticity of substitution of the search technology. It turns out, even though it is not directly visible, that condition (21) depends crucially on the feature of the urn-ball search technology assumed in Shi (2001). In particular, the right-hand side (RHS) will look different when the search technology is not urn-ball. A simple example is the case of CES where the RHS is a constant.

Recall that our condition (14) gives a condition for PAM for a given type distribution. To see that condition (21) arises as a special case of this, we now derive the equilibrium conditions in Shi (2001) with free entry and for a general search technology. Equilibrium profits can be obtained by substituting (6) into (5). If, after entry, seller type $y$ trades with buyer type $\mu(y)$ at queue length $\Lambda(y)$ (more precisely, $\Lambda(y, p(y))$ ), the free entry condition requires

$$
\left[m(\Lambda(y))-\Lambda(y) m^{\prime}(\Lambda(y))\right] f(\mu(y), y)=C(y) .
$$

Differentiating (22), after eliminating terms that add to zero by (7), and using the derivative of (6), we obtain that $m(\Lambda(y)) f_{y}(\mu(y), y)=C_{y}(y)$. For the special case of the urn-ball search technology $m_{1}$, these two equations coincide with Shi's (2001) characteristic equations. We can invert these to obtain an analytic expression of $\Lambda(y)$ as a function of the entry cost, and substitution into the RHS of (14) recovers Shi's (2001) result. ${ }^{14}$ Still the right-hand side of (14) depends crucially on the elasticity of substitution for the specific search

\footnotetext{
${ }^{14}$ For $m_{1}(\lambda)=1-e^{-\lambda}$, we obtain a nice analytic expression for the elasticity of substitution: $a(\Lambda)=\lambda^{-1}+e^{-\lambda} /\left(1-e^{-\lambda}\right)$. There are a multitude of ways to use the entry cost to substitute out the queue length along the equilibrium path. Observe that $m_{1}(\Lambda(y)) f_{y}(\mu(y), y)=C_{y}(y)$ implies $\Lambda(y)=-\ln \left(1-C_{y}(y) / f_{y}(\mu(y), y)\right)$. Using this, one could write the elasticity of substitution
} 
technology in question, as can easily be seen when the RHS of (14) is constant and, therefore, the level of entry plays no role. For urn-ball, the elasticity of substitution is nonconstant and indirectly depends on the entry cost. By varying the entry cost, any seller type distribution can be sustained (by setting the entry cost equal to the equilibrium profits) and by Proposition 3, square-rootsupermodularity provides the relevant bound on the strength of (21).

In our setting, entry does not simplify the analysis because inverting the free entry conditions yields $\Lambda(y)$ as a function of the inverse of the search technology, which for general search technologies does not have a nice analytic representation. Our approach, therefore, relies directly on the second-order conditions of the seller's optimization problem (4). Using a general search technology allows us to derive the fundamental economic trade-off between complementarities in match value and complementarities in the search technology, and to obtain explicit bounds on the strength of supermodularity that hold for any type distribution.

\subsection{Random Search}

In the Introduction, we compared our root-supermodularity condition to the conditions in the random search model of Shimer and Smith (2000). It is worth noting first that random search models adopt a notion of positive assortative matching that differs from the notion in this paper and in the frictionless environment of Becker (1973). In random search, sellers meet many different buyer types and the probability of meeting any particular buyer type is zero. Therefore, sellers are willing to accept matches from some set of buyer types. For a given seller, the set of buyers for which matching is mutually agreeable is then called the matching set. Positive assortative matching means that any element in the acceptance set of a lower type is either included or strictly below any element in the acceptance set of a higher type.

The conditions in Shimer and Smith (2000) derived their economic meaning from the fact that they ensure connectedness of these matching sets. The exact conditions are supermodularity of $f, \log$-supermodularity of $f_{x}$ and $f_{y}$, and log-supermodularity of $f_{x y}$. Unlike our match value function, theirs is a symmetric function $f$ such that $f(x, y)=f(y, x)$. They also assumed that $f \geq 0$ and $f_{y}(0, y) \leq 0 \leq f_{y}(1, y)$ for all $y$. These assumptions do not directly include log-supermodularity of $f$, which we used as a lower bound to compare the strength of our condition to theirs. We now show that log-supermodularity is

and thus the RHS of $(21)$ as $a(\Lambda(y))=-\ln \left(1-C_{y}(y) / f_{y}(\mu(y), y)\right)^{-1}+1-f_{y}(\mu(y), y) / C_{y}(y)$. Alternatively, one could use both entry conditions to express the elasticity of substitution as

$$
a(\Lambda(y))=\frac{C(y) f_{y}(\mu(y), y)\left[f_{y}(\mu(y), y)-C_{y}(y)\right]}{C_{y}(y)\left[f(\mu(y), y) C_{y}(y)-C(y) f_{y}(\mu(y), y)\right]},
$$

which exactly recovers the RHS of (21). 
implied under the additional monotonicity restriction imposed in our model, that is, $f_{y}(x, y) \geq 0$ (and by symmetry, $f_{x}(x, y) \geq 0$ ).

Assume that the conditions of the previous paragraph hold. A function $f$ is $\log$-supermodular if $\log f$ is supermodular or, equivalently, if for all $(x, y)$, the condition $f_{x y} f-f_{x} f_{y} \geq 0$ holds (where we suppress the arguments). Obviously this condition holds whenever $f_{x}=0$ because of supermodularity $\left(f_{x y} \geq 0\right)$ and $f \geq 0$. Now we establish that it holds even at points with $f_{x}>0$. First, observe that log-supermodularity trivially holds at $(0,0)$ under the assumptions above. Then it is sufficient to show that at any $(x, y)$ at which logsupermodularity holds, the left-hand side of the condition increases in $x$. The argument applies symmetrically for increases in $y$, which establishes the result that log-supermodularity holds at all $(x, y)$. The left-hand side of the logsupermodularity condition increases in $x$ if

$$
f_{x^{2} y} f+f_{x y} f_{x}-f_{x^{2}} f_{y}-f_{x} f_{x y} \geq 0 .
$$

Log-supermodularity of $f_{x}$ was assumed, which implies $f_{x^{2} y} f_{x}-f_{x^{2}} f_{x y} \geq 0$. From this inequality, we can now substitute for $f_{x^{2} y}$ in (23) and also substitute for $f_{x y}$ from the inequality of the log-supermodularity condition to get the more demanding inequality $f_{x^{2}} f_{y}+f_{x y} f_{x}-f_{x^{2}} f_{y}-f_{x} f_{x y} \geq 0$, which holds trivially.

We have, therefore, established that the conditions in Shimer and Smith (2000) together with monotonicity imply log-supermodularity. Although the reverse is not true (not every log-supermodular function fulfills the conditions in Shimer and Smith (2000); not all log-supermodular functions also have first and cross-partial derivatives that are log-supermodular), at least this result gives us a useful lower bound for the strength of supermodularity required under random search that can be used for comparison with our setting.

\subsection{Vanishing Frictions and Convergence to the Walrasian Equilibrium}

The competitive benchmark of the Walrasian economy (Becker (1973), Rosen (1974)) induces positive sorting under mere supermodularity. There are no frictions in a competitive setting. Such a lack of frictions can be captured in our setup by assuming that agents can perfectly match into pairs. This leads to a benchmark search technology represented by $m_{B}(\lambda)=\min \{\lambda, 1\}$ (see the kinked, solid line $m(\lambda)$ in Figure 1). The short side of the market always matches with probability 1 while those types on the long side get rationed in proportion to the buyer-seller ratio. We can now consider vanishing frictions to be a sequence of matching functions that converges to $m_{B}$ and investigate whether the condition for sorting reduces to mere supermodularity as required in the Walrasian benchmark.

This approach of considering the limit economy as frictions vanish ties in with the large literature that validates Walrasian trade as the limit of matching and bargaining games (see, among many others, Rubinstein and Wolinsky 


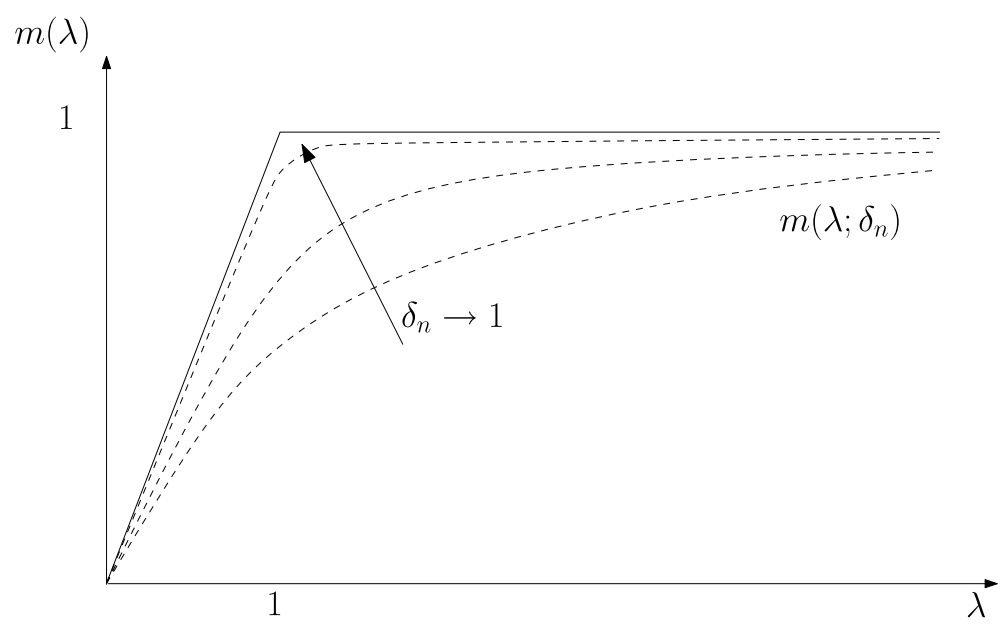

FIGURE 1.-Vanishing frictions for the static search technology.

(1985), Gale (1986), and more recently, Lauermann (2007)). This literature generally studies dynamic games and shows convergence as trading becomes more frequent. While this approach can be replicated with similar success in a dynamic extension of our setting, ${ }^{15}$ our contribution here is to take a different perspective by modeling vanishing frictions directly through changes in the search technology.

We obtain immediately an apparent discrepancy between the idea of convergence to Becker's (1973) supermodularity condition and the $n$-rootsupermodularity condition as implied by Theorem 1 . For example, the class of logarithmic search technologies $m(\lambda)=1-\ln \left(1+e^{(1-\lambda) /(1-\delta)}\right) / \ln \left(1+e^{1 /(1-\delta)}\right)$ with $\delta \in(0,1)$ fulfills the premise of Proposition 3 and, therefore, requires square-root-supermodularity for any level of $\delta$ to induce assortative matching. Yet it converges uniformly to the competitive benchmark $m_{B}(\lambda)$ as $\delta \rightarrow 1$, where we would expect the weaker condition of supermodularity (Becker (1973)) to apply.

To resolve this apparent discrepancy, observe that our condition for sorting entails the elasticity of substitution $a(\lambda, \delta)$ that depends on the search technology through the parameter $\delta .{ }^{16}$ While $m \rightarrow m_{B}$ uniformly as $\delta \rightarrow 1$, the elasticity of substitution does not converge to zero uniformly. In particular, in markets with few buyers, the elasticity of substitution remains close to one-

\footnotetext{
${ }^{15}$ The working paper version of this paper incorporates the fully dynamic extension of the model, including results on the convergence of our condition. We further discuss the dynamic model in the Conclusion.

${ }^{16}$ Some algebra establishes that $a(\Lambda, \delta)=\left(1+\exp \left(\frac{1-\Lambda}{1-\delta}\right)\right) \frac{1-\delta}{\Lambda}-\exp \left(\frac{1-\Lambda}{1-\delta}\right)(\ln (1+\exp (1 /(1-$

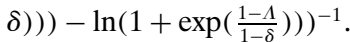


half. With vanishing frictions, the strength of the square-root-supermodularity condition comes only from the submarkets with few buyers $(\lambda \approx 0)$, that is, when at least some sellers match with very low probabilities due, for example, to an aggregate imbalance where the overall mass of sellers exceeds the mass of buyers. If this is not the case, that is, if all sellers can trade with probability bounded away from zero along a sequence of $\delta$ 's such that $m \rightarrow m_{B}$, then the standard supermodularity condition emerges: some tedious application of l'Hôpital's rule reveals that $\lim _{\delta \rightarrow 1} a(\lambda, \delta)=0$ for all $\lambda>0$. More generally this means that the set of seller types that trade with positive probability but for whom Becker's condition does not (approximately) govern the matching pattern includes only those sellers with queue length around zero (i.e., those that can hardly trade) as frictions vanish. Becker's (1973) insight is, therefore, recovered for vanishing frictions as it applies to all types that have nonvanishing trading prospects.

A special case is that of the CES search technology, because the only way to get convergence to $m_{B}$ is by changing the elasticity of substitution $a \rightarrow 0$. By construction, there is then not only uniform convergence of $m$, but also uniform convergence of $a$, and as a result, the necessary and sufficient condition for PAM converges to mere supermodularity for all matched pairs.

\section{CONCLUSION}

In the presence of search frictions in a market with two-sided matching, price competition gives rise to two distinct and opposing forces that determine sorting. The degree of complementarity in the match value is a force toward positive assortative matching, whereas search frictions embody a force toward negative assortative matching. We identify a condition based on the elasticities of substitution of the match value function and that of the search technology that summarizes this trade-off. It tells us exactly how much additional complementarity above and beyond mere supermodularity-namely root-supermodularity-is needed in terms of the match value to induce positive sorting, where the exact root depends on the elasticity of substitution in the search technology.

This elasticity condition also augments the standard Hosios (1990) condition for efficiency by relating different submarkets. In addition to the split of the surplus for a given pair of buyer-seller types as analyzed by Hosios, the novel determinant of efficiency here is which types are matched in equilibrium. Then not only is the derivative of the aggregate search technology important (as in Hosios), but also is the elasticity of substitution across different pairs.

In this work, we have made various simplifying assumptions. Some of them we relaxed in the working paper version of this paper. If seller preferences depend on the price and additionally their own type-for example, due to opportunity costs that depend on the seller's own type-our results still obtain, only now the match value is the sum of the buyer's and the seller's valuation: If the 
sellers' preferences are of the form $f^{s}(y)+p$, then our conditions on the match value function refer to $f(x, y)+f^{s}(y)$. Our results further generalize if sellers also care about the buyer's type, provided they are able to specify the desired buyer type together with the price so as to avoid problems of adverse selection. Alternatively, our results apply if the seller posts a payoff he wants to obtain (rather than a price), which makes the buyer the residual claimant. In addition to the preferences, we also relax the time structure. We consider steady states in a repeated interaction and show that $n$-root-supermodularity still ensures positive assortative matching. The condition of $n$-root-supermodularity $\left(n=1-\bar{a}^{-1}\right)$ is still sufficient, though a weaker root that depends on the discount factor may also suffice. ${ }^{17}$

We conclude with a final thought on the connection to many-to-many matching markets for which the literature yet lacks a characterization of the sorting patterns. While our setup requires each seller to trade a single unit with at most one buyer, it does resemble a particular kind of two-sided many-to-many matching market. When $\beta$ buyers of type $x$ and $\sigma$ sellers of type $y$ form a coalition, they produce output $M(\beta, \sigma) f(x, y)$. Instead of buyers and sellers, the sides can be interpreted as teachers and students, where a coalition is a school, or machines and workers, where a coalition is a factory. Given the similarity in structure, we expect our results to apply to this setting as well.

\section{APPENDIX}

PROOF OF THEOREM 1: We prove the result for case (i), positive assortative matching. An analogous derivation establishes the result for negative assortative matching. The proof for PAM consists of two parts, one for the sufficient condition, and one for the necessary condition.

Proposition A1-Sufficiency: If the function $f(x, y)$ is strictly n-rootsupermodular where $n=(1-\bar{a})^{-1}$, then any equilibrium entails positive assortative matching under any type distributions $B(x), S(y)$.

ProOF-By Contradiction: Consider a (candidate) equilibrium $(G, H)$ that does not entail positive assortative matching. Then there exist $(x, y, p)$ and $\left(x^{\prime}, y^{\prime}, p^{\prime}\right)$ on the support of $H$ such that $x>x^{\prime}$ but $y<y^{\prime}$. Then $x$ has to be part of the solution to the seller's optimization problem (4) for $y$, and $x^{\prime}$ has to be part of the solution to (4) for $y^{\prime}$, given $U(\cdot, G, H)$. We contradict this in four steps.

\footnotetext{
${ }^{17}$ For the search technologies in Proposition 3, square-root-supermodularity remains necessary, while for CES matching technologies, weaker conditions apply that depend on the discount factor. Note that these results assume the existence of a steady state, which can be assured under a "cloning" assumption that we make in the working paper.
} 
Step 1-Reformulating the sellers' maximization problem. The optimization problem (4) of seller $y$ can be written as

$$
\begin{gathered}
\max _{x, \lambda, p}\{m(\lambda) p: q(\lambda)[f(x, y)-p]=U(x, G, H)\} \\
\quad \Leftrightarrow \quad \max _{x, \lambda}\{m(\lambda) f(x, y)-\lambda U(x, G, H)\} \\
\quad \Leftrightarrow \quad \max _{x} \Pi(x, y \mid U(\cdot, G, H)),
\end{gathered}
$$

where $\Pi$ in the last line is defined as

$$
\Pi(x, y \mid V(\cdot)) \equiv \max _{\lambda} m(\lambda) f(x, y)-\lambda V(x)
$$

for any positive and continuous function $V(\cdot)$. The following obvious property will be useful later:

(I) For any two positive and continuous functions $V(\cdot)$ and $W(\cdot)$, and any seller type $x$, the inequality $\Pi(x, y \mid V(\cdot))<\Pi(x, y \mid W(\cdot))$ holds if and only if $V(x)>W(x)$.

We have achieved the desired contradiction if the maximizer of (24) for $y$ is smaller than for $y^{\prime}$. Defining $\Gamma(y \mid V(\cdot))=\arg \max _{x} \Pi(x, y \mid V(\cdot))$, this means that we have achieved the contradiction if

$$
\max \Gamma(y \mid U(\cdot, G, H)) \leq \min \Gamma\left(y^{\prime} \mid U(\cdot, G, H)\right) .
$$

Step 2-Introducing differentiability through auxiliary buyer utility $V(\cdot)$. To show (26), it will convenient to have $\Pi$ differentiable. To achieve this, we do not directly work with buyers' equilibrium utility $U(\cdot, G, H)$, but rather we work with a particular auxiliary function $V(\cdot)$ that we define implicitly as

$$
\Pi(x, y \mid V(\cdot))=\Pi(\varkappa, y \mid U(\cdot, G, H))
$$

for all $x \leq \varkappa \equiv \max \Gamma(y \mid U(\cdot, G, H))$, and $V(x)=U(x, G, H)$ otherwise. This means that if seller $y$ has to leave utility $V(x)$ to the buyers, he is indifferent between all types that are below $\varkappa$, that is, $\Gamma(y \mid V)=[\underline{x}, \varkappa]$. Equation (27) defines $V(x)$ uniquely by property (I) established in the previous step. Note that $V(x)$ is differentiable by construction since the implicit function theorem delivers

$$
V^{\prime}(x)=\frac{m(\lambda)}{\lambda} f_{x}(x, y),
$$

where $\lambda$ takes the value that maximizes the right-hand side of (25). Since $\varkappa$ is a maximizer of $\Pi(x, y \mid U(\cdot, G, H))$, property (I) also establishes another property: (II) $V(x) \leq U(x, G, H)$ everywhere.

Step 3-Positive cross-partials. Now consider seller $y^{\prime}>y$ in a neighborhood of $y$. Taking the cross-partial of $\Pi\left(x, y^{\prime} \mid V\right)$ and incorporating that $V$ is defined 
by (27) together with (25), we obtain, after some tedious algebra, for all $x \in$ $[\underline{x}, \varkappa]$ that

$$
\begin{aligned}
& \left.\frac{\partial \Pi\left(x, y^{\prime} \mid V\right)}{\partial x \partial y^{\prime}}\right|_{y^{\prime}=y} \\
& =\left[f_{x y}(x, y)-\frac{m^{\prime}(\lambda)\left(\lambda m^{\prime}(\lambda)-m(\lambda)\right)}{\lambda m^{\prime \prime}(\lambda) m(\lambda)} \frac{f_{x}(x, y) f_{y}(x, y)}{f(x, y)}\right] m(\lambda),
\end{aligned}
$$

where $\lambda$ takes the value that maximizes the right-hand side of (25). This crosspartial evaluated at $y^{\prime}=y$ is strictly positive since the RHS of (28) is strictly positive by strict $n$-root-supermodularity of $f$. Hence for $y^{\prime}$ slightly larger than $y$, the cross-partial remains strictly positive by continuity. On $[\underline{x}, x]$ we have $\Pi(x, y \mid V)=\Pi\left(x^{\prime}, y \mid V\right)$ by construction and, therefore, $\Pi\left(x, y^{\prime} \mid V\right)<$ $\Pi\left(x^{\prime}, y^{\prime} \mid V\right)$ when $x<x^{\prime}$. Therefore, any $x$ that maximizes $\Pi$ has to lie above $\varkappa$, and we obtain a third property: (III) $\min \Gamma\left(y^{\prime} \mid V\right) \geq \varkappa$.

Step 4-Reintroducing $U(\cdot, G, H)$ instead of the auxiliary buyer utility $V(\cdot)$. By construction, $V(x)=U(x, G, H)$ for $x \geq \varkappa$ and, by (II), it holds that $V(x) \leq U(x, G, H)$ everywhere. Therefore, by (I) we have $\Pi(x, y \mid V)=$ $\Pi(x, y \mid U(\cdot, G, H))$ for $x \geq \varkappa$ and $\Pi(x, y \mid V) \geq \Pi(x, y \mid U(\cdot, G, H))$ everywhere. Since by (III), $\min \Gamma\left(y^{\prime} \mid V\right) \geq \varkappa$, this implies immediately that $\min \Gamma\left(y^{\prime} \mid\right.$ $U(\cdot, G, H)) \geq \varkappa$. By the definition of $\varkappa$, this implies (26).

Q.E.D.

PROPOSITION A2 - Necessity: If any equilibrium is positive assorted under any type distributions $B(x)$ and $S(y)$, then $f(x, y)$ is weakly n-root-supermodular where $n=(1-\bar{a})^{-1}$.

PROOF: By contradiction. Suppose there exists some $(\hat{x}, \hat{y})$, such that the match value function is not $n$-root-supermodular, but there exists an equilibrium that exhibits PAM for any distributions $B, S$. We will contradict this in four steps; the main insights are in the first three steps.

Step $1-$ Construct a set $Z_{\varepsilon}$ around $(\hat{x}, \hat{y})$, where $f$ is nowhere n-rootsupermodular. By the smoothness properties of $f$, there exists $\varepsilon>0$ such that $f$ is not root-supermodular anywhere on $Z_{\varepsilon}=[\hat{x}-\varepsilon, \hat{x}+\varepsilon] \times[\hat{y}-\varepsilon, \hat{y}+\varepsilon]$. We can choose $\varepsilon$ such that

$$
f_{x y}(x, y)-\alpha \frac{f_{x}(x, y) f_{y}(x, y)}{f(x, y)}<0
$$

for all $(x, y) \in Z_{\varepsilon}$ for some $\alpha<\bar{a}$. By continuity of $a(\lambda)$, there exists $\lambda_{1}, \lambda_{2}$ such that $a(\lambda)>\alpha$ for all $\lambda \in\left[\lambda_{1}, \lambda_{2}\right]$. If buyer and seller types are in $Z_{\varepsilon}$ and they trade at queue lengths in $\left[\lambda_{1}, \lambda_{2}\right]$, the lack of sufficient supermodularity means that PAM cannot be sustained, as we formalize in the next steps.

Step 2-Let $Z_{\varepsilon}$ shrink so that types are similar. Consider a sequence $\left\{\varepsilon_{k}\right\}_{k=1}^{\infty}$, $0<\varepsilon_{k}<\varepsilon$, that monotonically converges to zero. Let $B_{k}$ and $S_{k}$ be associated 
sequences of distributions of buyer and seller types. Let $B_{k}$ be uniform with support on $\mathcal{X}_{k}=\left[\hat{x}-\varepsilon_{k}, \hat{x}+\varepsilon_{k}\right]$ and unit mass $B_{k}\left(\hat{x}+\varepsilon_{k}\right)=1$. Let $S_{k}$ be uniform with support on $\mathcal{Y}_{k}=\left[\hat{y}-\varepsilon_{k}, \hat{y}+\varepsilon_{k}\right]$ with mass $S_{k}\left(\hat{y}+\varepsilon_{k}\right)=2 /\left(\lambda_{1}+\right.$ $\lambda_{2}$ ), that is, the aggregate ratio of buyers to sellers remains constant at the average of $\lambda_{1}$ and $\lambda_{2}$, independent of $k$. By construction, the buyer-seller types that trade are within $Z_{\varepsilon}$ for any $k$.

Step 3-For some $k$, all buyers and sellers trade at queue lengths in $\left(\lambda_{1}, \lambda_{2}\right)$. Consider an equilibrium $\left(G_{k}, H_{k}\right)$ for each $k$. Note first that the difference in expected buyer utilities converges to zero, in the sense that for every $\xi>0$, there exists $\kappa$ such that $\left|U\left(x_{1}, G_{k}, H_{k}\right)-U\left(x_{2}, G_{k}, H_{k}\right)\right| \leq \xi$ for any $x_{1}, x_{2} \in \mathcal{X}_{k}$ and any $k \geq \kappa$. This notion of convergence is used throughout this proof. It can be shown based on equilibrium condition (ii), which ensures that $\left|U\left(x_{1}, G_{k}, H_{k}\right)-U\left(x_{2}, G_{k}, H_{k}\right)\right| \leq \max _{\lambda \in[0, \infty]} \max _{y \in \mathcal{Y}_{k}} q(\lambda) \mid f\left(x_{1}, y\right)-$ $f\left(x_{2}, y\right) \mid$. Assuming without loss of generality that $x_{1} \geq x_{2}$, the right-hand side of the inequality is bounded by $f\left(x_{1}, \hat{y}+\varepsilon_{k}\right)-f\left(x_{2}, \hat{y}-\varepsilon_{k}\right)$, and this term vanishes since $x_{1}-x_{2} \leq 2 \varepsilon_{k} \rightarrow 0$ and $\hat{y}+\varepsilon_{k}-\left(\hat{y}-\varepsilon_{k}\right)=2 \varepsilon_{k} \rightarrow 0$ and $f$ is continuous. Given that the differences in buyer utility vanish with large $k$ and given that the distance in types vanishes, it is easy to show that the distance between the highest queue length that is part of a solution to (4) for some $y \in \mathcal{Y}_{k}$ and the lowest queue length that is part of a solution to (4) for some $y^{\prime} \in \mathcal{Y}_{k}$ converge to zero. (Also the differences in the value to program (4) across seller types in $\mathcal{Y}_{k}$ vanish with increasing $k$, as used in the next step.) Since the differences in queue lengths across sellers vanish, but the aggregate buyer seller ratio is $\left(\lambda_{1}+\lambda_{2}\right) / 2$, all sellers trade at queue lengths in $\left(\lambda_{1}, \lambda_{2}\right)$ for $k$ sufficiently large. If we restrict attention only to differentiable equilibria, this immediately contradicts the assumption that the equilibria are PAM, since condition (14) in Lemma 1 is violated.

Step 4-Nondifferentiable equilibria. Finally, we rule out that equilibria are PAM but nondifferentiable. Let $\pi_{k}(y)=\max _{p} \pi\left(y, p, G_{k}, H_{k}\right)$ denote the equilibrium profit of seller $y$, that is, the value of program (4). In the previous proof of Proposition A1, the indifference condition (27) which defines auxiliary utility $V_{k}(x)$ can be restated as $\Pi\left(x, y \mid V_{k}(\cdot)\right)=\pi_{k}(y)$ or

$$
\max _{\lambda} m(\lambda) f(x, y)-\lambda V_{k}(x)=\pi_{k}(y) .
$$

Note that the maximizer of the left-hand side (LHS) of (29) is used in (28) in the previous proof. We are done if we can show that there exists $k$ such that the maximizers of the LHS of (29) lie in $\left[\lambda_{1}, \lambda_{2}\right]$ for all $x \in \mathcal{X}_{k}$ and any $y \in \mathcal{Y}_{k}$. Then analogous arguments as in the proof of Proposition A1 establish that there has to be negative assortative matching since the cross-partial in (28) is negative, ruling out PAM.

To show the missing part, recall that the equilibrium profits $\pi_{k}(y)$ across sellers in $\mathcal{Y}_{k}$ becomes nearly identical for large $k$ (see previous Step 3). Since profits lie in a bounded set, there exist limit point $\pi_{\infty}$ and a subsequence such 
that for any $\xi$, the distance between the equilibrium profit $\pi_{k}(y)$ of any $y \in \mathcal{Y}_{k}$ and $\pi_{\infty}$ are less than $\xi$, as $k$ becomes sufficiently large. This convergence of the RHS of (29) and the vanishing differences between buyer types mean that there is a subsequence for which $V_{k}(x)$ approaches some limit value $V_{\infty}$ arbitrarily close for all $x \in \mathcal{X}_{k}$ and any $y \in \mathcal{Y}_{k}$. Since $V_{k}(x)$ converges to $V_{\infty}$ and the support of buyer types shrinks to $\hat{x}$, the queue lengths that maximize the LHS of (29) have to converge. Finally, observe that they have to converge to a value within $\left[\lambda_{1}, \lambda_{2}\right]$ as we will now show. The profit $\pi_{k}(y)$ can by (5) be written as $\max _{x, \lambda} m(\lambda) f(x, y)-\lambda U\left(x, G_{k}, H_{k}\right)$. Let $\left(x_{k}^{*}, \lambda_{k}^{*}\right)$ be the equilibrium type and equilibrium queue length which maximize this expression. Since equilibrium queue lengths lie in $\left[\lambda_{1}, \lambda_{2}\right]$ for large $k$ as shown in Step 3, we have $\lambda_{k}^{*} \in\left(\lambda_{1}, \lambda_{2}\right)$ for $k$ large enough. Since all maximizers of the LHS of (29) converge, and $\lambda_{k}^{*}$ is such a maximizer (for $x_{k}^{*}$ ), all maximizers converge to the limit of $\lambda_{k}^{*}$ that lies within $\left(\lambda_{1}, \lambda_{2}\right)$.

Q.E.D.

Proof of Proposition 1: (i) Given search technology $m$, let $a_{1}=\frac{1}{3} \bar{a}+\frac{2}{3} \underline{a}$ and $a_{2}=\frac{2}{3} \bar{a}+\frac{1}{3} \underline{a}$. Choose $\lambda_{1}$ and $\lambda_{2}$ such that $a(\lambda) \in\left[a_{1}, a_{2}\right]$ for all $\lambda \in\left[\lambda_{1}, \lambda_{2}\right]$. Consider $f(x, y)=(x+y+1)^{\left(n+n_{2}\right) / 2}$. This function is $n_{2}$-root-supermodular but nowhere $n$-root-supermodular, where $n_{2}=\left(1-a_{2}\right)^{-1}$ and $n=(1-\underline{a})^{-1}$. Now consider a sequence of distributions $B_{k}$ and $S_{k}$ with support on $\left[0, \varepsilon_{k}\right]$, with $B_{k}\left(\varepsilon_{k}\right)=1$ and $S_{k}\left(\varepsilon_{k}\right)=2 /\left(\lambda_{1}+\lambda_{2}\right)$. Analogous arguments as in Steps 24 in the proof of Proposition A2 show that all agents desire trade at a queue lengths in $\left(\lambda_{1}, \lambda_{2}\right)$, and $n_{2}$-root-supermodularity implies PAM. This establishes the first part. Part (ii) can be established analogously, with preference function $f(x, y)=(x+y+1)^{\left(n+n_{1}\right) / 2}$, where $n_{1}=\left(1-a_{1}\right)^{-1}$ and $n=(1-\underline{a})^{-1}$. Q.E.D.

PROOF OF PROPOSITION 2: We prove the result for positive sorting; the proof for negative sorting is analogous. We construct a positively assorted differentiable equilibrium $(G, H)$ in three steps: First we explore necessary conditions that restrict the connection between the queue length, the assignment, and the price that different seller types face in equilibrium. Then we "reverseengineer" the associated equilibrium $(G, H)$, and, finally, we check that the equilibrium conditions are indeed met.

Step 1-Exploiting necessary conditions. Rather than consider equilibrium distributions $(G, H)$ directly, we reverse-engineer them by exploiting first some necessary conditions about the relationship between the queue length $\Lambda(y)$ [formally $\Lambda(y, p(y))$ ], the assignment $\mu(y)$, and the price $p(y)$ in a differentiable equilibrium.

First, the buyer-seller ratio integrated over a range of seller types equals the number of buyers that choose these types (as required by the Radon-Nikodym derivative), which relates $\Lambda$ to $\mu$ via $\int_{y}^{\bar{y}} \Lambda(\cdot) d S=\int_{\mu(y)}^{\bar{x}} d B$. This yields

$$
\mu^{\prime}(y)=s(y) \Lambda(y) b(\mu(y))^{-1} .
$$


Second, $\Lambda$ and $\mu$ are linked via the first-order conditions given in (6) and (7) for some positive and increasing function $U(y)$. From (6) and (7) we can derive (11), which together with (30) yields

$$
\begin{aligned}
\Lambda^{\prime}(y)= & -\frac{1}{m^{\prime \prime}(\Lambda(y)) f(\mu(y), y)} \\
& \times\left[\frac{\left(\Lambda(y) m^{\prime}(\Lambda(y))-m(\Lambda(y))\right) s(y)}{b(\mu(y))} f_{x}(\mu(y), y)\right. \\
& \left.+m^{\prime}(\Lambda(y)) f_{y}(\mu, y)\right] .
\end{aligned}
$$

Third, $\Lambda$ and $\mu$ are linked via two boundary conditions. Intuitively, the lowest active seller type, that is, the lowest type $x_{0}$ that does not take the outside option, has to obtain at least as much utility as the outside option of zero and has to get exactly zero if $x_{0}>\underline{x}$; otherwise, lower types would get more by becoming active. A similar logic holds for the lowest seller type $y_{0}$ that trades in equilibrium. Therefore, the boundary buyers' equilibrium utility [given in (6)] and the boundary sellers' equilibrium profits [given by (6) substituted into (5)] have to satisfy

$$
\begin{aligned}
& m^{\prime}\left(\Lambda\left(y_{0}\right)\right) f\left(\mu\left(y_{0}\right), y_{0}\right) \geq 0 \quad \text { with equality if } \mu\left(y_{0}\right)>\underline{x}, \\
& {\left[m\left(\Lambda\left(y_{0}\right)\right)-\Lambda\left(y_{0}\right) m^{\prime}\left(\Lambda\left(y_{0}\right)\right)\right] f\left(\mu\left(y_{0}\right), y_{0}\right) \geq 0} \\
& \quad \text { with equality if } y_{0}>\underline{y} .
\end{aligned}
$$

Equations (30) and (31) together constitute a differential equation system in $\Lambda, \mu$. One initial condition is $\mu(\bar{y})=\bar{x}$. Given a second initial condition on the queue length at the top seller, $\Lambda(\bar{y})=\bar{\lambda} \in(0, \infty)$, the system uniquely determines $\Lambda(y)$ and $\mu(y)$ (in the direction of lower $y$ ) at all $y$ down to some limit point $y_{0}(\bar{\lambda})$. This limit point is characterized either by $y_{0}(\bar{\lambda})=\underline{y}$, or by $\mu\left(y_{0}(\bar{\lambda})\right)=\underline{x}$, or by $\lim _{y \backslash y_{0}(\bar{\lambda})} \Lambda(y)=0$, or by $\lim _{y \backslash y_{0}(\bar{\lambda})} \Lambda(y)=\infty$, whichever arises first. Since the lower bound has to satisfy (32) and (33), this imposes restrictions on the free parameter $\bar{\lambda}$. We can show (the proof is available in the working paper version of the paper) that there exists an initial condition $\bar{\lambda} \epsilon$ $(0, \infty)$ such that the resulting $y_{0}(\bar{\lambda}), \lambda\left(y_{0}(\bar{\lambda})\right)$, and $\mu\left(y_{0}(\bar{\lambda})\right)$ fulfill boundary conditions (32) and (33). For the following discussion, consider such a $\bar{\lambda}$, which fixes the associated solutions $\Lambda$ and $\mu$ to (30) and (31), and fixes associated boundary types $y_{0}$ and $x_{0}=\mu\left(y_{0}\right)$ uniquely.

The price function $p(y)$ for each type $y \geq y_{0}$ can then be reconstructed since the profit $m(\Lambda(y)) p(y)$ has to equal the constructed profits given by (6) substituted into (5), yielding after division by $m(\Lambda(y))$ that $p(y)=$ $\left[1-\Lambda(y) m^{\prime}(\Lambda(y)) / m(\Lambda(y))\right] f(\mu(y), y)$. For types below $y_{0}$, note that $y_{0}>$ 
$y$ implies by (32) that $\Lambda\left(y_{0}\right)=\lim _{y \rightarrow y_{0}} \lambda(y)=0$, which implies $p\left(y_{0}\right)=$ $\overline{\lim }_{y \rightarrow y_{0}} p(y)=0$ since $\lim _{\Lambda \rightarrow 0} m(\Lambda) / \Lambda=m^{\prime}(0)$ and, therefore, $\lim _{y \rightarrow y_{0}} \Lambda(y) \times$ $m^{\prime}(\Lambda(y)) / m(\Lambda(y))=1$. Note that the finite $\operatorname{limit}_{\lim _{\Lambda \rightarrow 0} m(\Lambda) / \Lambda=m^{\prime}(0)}$ indeed exists: finite $m^{\prime}(\lambda)$ exists by assumption for $\lambda>0$, is monotone (by $m^{\prime \prime}(\lambda)<0$ ), and is bounded (by $m^{\prime}(\lambda) \leq 1$, as otherwise $\lim _{\lambda \rightarrow 0} q(\lambda)=$ $\lim _{\lambda \rightarrow 0} m(\lambda) / \lambda=\lim _{\lambda \rightarrow 0} m^{\prime}(\lambda)>1$, which violates $\left.q(\lambda) \in[0,1]\right)$. The boundary seller does not obtain any buyers even at a zero price, and all types below him also obtain no buyers independent of the price they charge because their quality is also too low. So we can set $p(y)=0$ for all $y<y_{0}$.

Step 2-Recovering the equilibrium $(G, H)$. The equilibrium distributions $(G, H)$ can now be constructed from the $\mu$ and $p$ functions derived in the first step. Consider the sellers first. We integrate all of them that offer prices below $p$ as derived in the previous step:

$$
G(y, p)=\int_{y_{0}}^{y} s(\tilde{y}) I_{[p(\tilde{y}) \leq p]} d \tilde{y},
$$

where $I$ is an indicator function that takes the value of 1 if the qualifier in square brackets it true and takes the value 0 otherwise. Clearly, $G_{\mathcal{Y}}=S$ by construction, as required. Next consider the buyers. Types below $x_{0}$ choose their outside option $\emptyset$. That is, at any price $p \geq 0$ these types trade below (by our convention in footnote 5) and, therefore, have mass $B(x)$. Therefore, for all $x<x_{0}$ we have $H(x, y, p)=B(x)$ for all $(y, p) \in(\mathcal{Y} \times \mathcal{P}) \cup\{\emptyset\}$. For all buyers with $x \geq x_{0}$ we have $H(x, \emptyset)=B\left(x_{0}\right)$ and for all other $(y, p) \in \mathcal{Y} \times \mathcal{P}$,

$$
H(x, y, p)=\int_{y_{0}}^{y} b(\mu(\tilde{y})) I_{[\mu(\tilde{y}) \leq x]} I_{[p(\tilde{y}) \leq p]} d \tilde{y}+B\left(x_{0}\right) .
$$

Clearly $H_{\mathcal{X}}=B$, as required.

Step 3-Checking the equilibrium conditions. By construction, the function $\Lambda(y)$ as constructed in the first step coincides with a Radon-Nikodym derivative $\Lambda_{G H}(y, p)$ of $G$ with respect to $H$ along all $(y, p(y))$. Also, the function $U(\cdot)$ in the first step coincides with $U(\cdot, G, H)$ by construction. To check that $(G, H)$ is indeed an equilibrium, we can extend $\Lambda_{G H}$ to the entire domain by (3) and check the equilibrium conditions (i) and (ii).

Condition (i) amounts to verifying that no seller $y$ wants to deviate and offer a different price than $p(y)$ constructed above (because $(y, p(y))$ are the only combinations in the support of $G$ ), which is equivalent to checking that no seller has a profitable deviation from $(\mu(y), \Lambda(y), p(y))$ in (4). Additionally, condition (ii) requires us to check that no buyer $\mu(y)$ wants to deviate and trade at some combination other than $(y, p(y))$ (again $(\mu(y), y, p(y))$ are the only combinations in the support of $H$, except for those buyers below $x_{0}$ for which we have to check that they do not want to trade at all).

The verification is facilitated by the observations that if sellers do not have an incentive to deviate, then buyers have no incentive to deviate. This follows 
directly from the fact that a profitable deviation for buyers means that, in program (4), sellers can make higher profits. (Another way to see this is that (31) is exactly the buyers' envelope condition.) Moreover, for the sellers, we only have to consider types in $\left[y_{0}, \bar{y}\right]$. If there are seller types below $y_{0}$, these types do not have a profitable deviation because, by boundary condition (33), type $y_{0}$ makes zero profits and we will verify that he does not have a profitable deviation despite being a higher type.

For types in $\left[y_{0}, \bar{y}\right]$ we know that $(\mu(y), \Lambda(y), p(y))$ constructed above is indeed a local maximum in (4), because $n$-root-supermodularity implies that the Hessian (8) is negative definite. We now establish that the solution is a global maximum. Consider a seller $y$ with assigned buyer type $x$, that is, $x=$ $\mu(y)$. Now suppose there is another buyer $x^{\prime}=\mu\left(y^{\prime}\right)$, different from $x$, which is optimal for $y$, that is, $x^{\prime}$ satisfies the necessary first-order conditions (6) and (7) for optimality for seller $y$ (together with for some queue length). ${ }^{18}$ Since $\left(x^{\prime}, y\right)$ fulfill both (6) and (7), they satisfy the generalization of $(6)$,

$$
q\left(s\left(x^{\prime}, y\right)\right) f_{x}\left(x^{\prime}, y\right)-U^{\prime}\left(x^{\prime}\right)=0,
$$

where $s\left(x^{\prime}, y\right)$ is defined as the queue length such that $\Lambda(y)=s\left(x^{\prime}, y\right)$ solves $m^{\prime}(\Lambda(y)) f\left(x^{\prime}, y\right)-U\left(x^{\prime}\right)=0$ in analogy to (7). Now suppose that $x^{\prime}>x$, which implies $y^{\prime}>y$; the opposite case is analogous. Since $\mu\left(y^{\prime}\right)=x^{\prime}$, these types also fulfill (6) and (7) by our construction in Step 1; therefore, they fulfill also

$$
q\left(s\left(x^{\prime}, y^{\prime}\right)\right) f_{x}\left(x^{\prime}, y^{\prime}\right)-U^{\prime}\left(x^{\prime}\right)=0 .
$$

We rule out that both (35) and (36) are satisfied simultaneously by showing that $q\left(s\left(x^{\prime}, y\right)\right) f_{x}\left(x^{\prime}, y\right)$ is strictly increasing in $y$. The derivative of this expression with respect to $y$, together with implicit differentiation of (7) to recover $\partial \varsigma\left(x^{\prime}, y\right) / \partial y$, is strictly positive if and only if

$$
f_{x y}\left(x^{\prime}, y\right)>a\left(s\left(x^{\prime}, y\right)\right) f_{y}\left(x^{\prime}, y\right) f_{x}\left(x^{\prime}, y\right) f\left(x^{\prime}, y\right)^{-1},
$$

which is ensured by $n$-root-supermodularity (where $\left.n=(1-\bar{a})^{-1}\right)$. This implies that the solution to the first-order condition in (6) and (7) is a global maximum.

Q.E.D.

Proof of Proposition 3: Trade in pairs requires $\lambda q(\lambda)=m(\lambda)$. Therefore, $q^{\prime \prime}(\lambda)=\left[m^{\prime \prime}(\lambda)-2 q^{\prime}(\lambda)\right] \lambda^{-1}$. Then $\left|q^{\prime \prime}(0)\right|<\infty$ implies $m^{\prime \prime}(0)=2 q^{\prime}(0)$. Together with $q^{\prime}(0) \neq 0$, this implies $m^{\prime \prime}(0) \neq 0$. Use $\lambda q(\lambda)=m(\lambda)$ to write (13) as $a(\lambda)=m^{\prime}(\lambda) q^{\prime}(\lambda) /\left(m^{\prime \prime}(\lambda) q(\lambda)\right)$ and substitute to get $a(0)=$ $m^{\prime}(0) /(2 q(0))$. Since $q(0)=\lim _{\lambda \rightarrow 0} m(\lambda) / \lambda=m^{\prime}(0)$, one obtains $a(0)=1 / 2$.

\footnotetext{
${ }^{18}$ This argument assumes $x^{\prime}$ satisfied $x^{\prime}=\mu\left(y^{\prime}\right)$ for some $y^{\prime}$, which does not hold if $x^{\prime}<x_{0}$. Note that in the case $x^{\prime}<x_{0}$, both types $x^{\prime}$ and $x_{0}$ obtain zero utility (see (32)), and seller $y$ is at least as well off according to (4) by attracting $x_{0}$ as by attracting $x^{\prime}$. For $x_{0}$ it holds that $\mu\left(y_{0}\right)=x_{0}$.
} 
Further, $a(\lambda) \leq 1 / 2$ for all $\lambda$ if and only if $q(\lambda)^{-1}$ is convex: since $q(\lambda)^{-1}=$ $\lambda m(\lambda)^{-1}$, we have (suppressing the argument of $\left.m\right)\left(\lambda m^{-1}\right)^{\prime \prime}=\lambda^{2} m^{-3}\left[-m^{\prime \prime} q+\right.$ $2 m^{\prime} q^{\prime}$ ]. This is positive if and only if $-m^{\prime \prime} q+2 m^{\prime} q^{\prime} \geq 0$ or, equivalently, $a(\lambda)=m^{\prime} q^{\prime} /\left(m^{\prime \prime} q\right) \leq 1 / 2$.

Q.E.D.

PROOF OF PROPOSITION 4: We first show that the planner's assignment coincides with the equilibrium assignment if it is positive assortative. Then we sketch why root-supermodularity implies the associated direction of sorting by showing that it induces the direction locally (the full proof for global assortative matching is available on request).

Assume that $H$ in the planner's solution is assortative, that is, it permits $\mu$ that is strictly monotone. Since $H_{\mathcal{X}}(\mu(y), y)=B(y)$ and $H_{\mathcal{X}}=B$, all the mass is concentrated only on $(\mu(y), y)$ pairs. For a given $(\mu(y), y)$ pair, the concavity of the matching function implies that it is optimal if all of these agents trade at the same queue length $\Lambda(y)$ [formally, $\Lambda(y, p(y))$ for some $p(y)] \cdot{ }^{19}$ Since all mass is only concentrated on $(\mu(y), y)$ pairs, the constraints can be conveniently summarized by a single constraint $\int_{y}^{\bar{y}} \Lambda(\cdot) d S=1-B(\mu(y))$ in case of positive sorting and $\int_{y}^{\bar{y}} \Lambda(\cdot) d S=B(\mu(y))$ in case of negative sorting. For given $(G, H)$, there is almost everywhere a unique $\Lambda$ fulfilling this constraint, and a given $\Lambda$ yields unique $\mu$ and, thus, a unique $(G, H)$ [for the given $p(y)$ ] as can be seen by the analogous construction in Step 2 of the existence proof. The planner can, therefore, directly control $\Lambda$, which by the constraint governs the assignment $\mu$, leading to the much simpler control problem

$$
\begin{gathered}
\max _{\Lambda, y_{0}} \int_{y_{0}}^{\bar{y}} s(y) \cdot m(\Lambda(y)) \cdot f(\mu(y), y) d y, \\
\text { s.t. } \quad \mu^{\prime}(y)= \pm s(y) \Lambda(y) / b(\mu(y)),
\end{gathered}
$$

where the sign on the constraint is positive for positive sorting and negative for negative sorting, $y_{0}$ denotes the lowest type that is assigned to buyers by the planner.

The Hamiltonian to problem (37) is:

$$
\mathcal{H}(y, \lambda, \mu)=s(y) \cdot m(\Lambda) \cdot f(\mu, y)+\phi \cdot s(y) \Lambda / b(\mu),
$$

where $\phi$ is the multiplier.

\footnotetext{
${ }^{19}$ Formally, the objective in (18) can be written as $\max _{G, H} \int q\left(\Lambda_{G H}(y, p)\right) f(\mu(y), y) d H_{\mathcal{Y P}}$, which is equivalent to $\max _{G, H} \int m\left(\Lambda_{G H}(y, p)\right) f(\mu(y), y) d G$ by the third constraint. This problem is equivalent to $\max _{G, H} \int m(\Lambda(y)) f(\mu(y), y) d G_{\mathcal{Y}}$, such that $G_{\mathcal{Y}}=S, H_{\mathcal{X}}=B$ and $\Lambda=$ $d H_{\mathcal{Y}} / d G_{\mathcal{Y}}$ where $\Lambda(y):=\int \Lambda_{G H}(y, p) d G(p \mid y)$, since the concavity of $m$ always makes it optimal to assign the average queue length.
} 
The optimality conditions of the Hamiltonian satisfy:

$$
\begin{aligned}
\Lambda: & \frac{\partial \mathcal{H}}{\partial \Lambda}=m^{\prime}(\Lambda) \cdot f(\mu, y)+\frac{\phi}{b(\mu)}=0, \\
\mu: & \frac{\partial \mathcal{H}}{\partial \mu}=s(y) \cdot m(\Lambda) \cdot f_{x}(\mu, y)-\phi \cdot s(y) \Lambda \frac{b^{\prime}(\mu)}{b^{2}(\mu)}=-\phi^{\prime} .
\end{aligned}
$$

Defining $A(\mu(y))=-\frac{\phi(y)}{b(\mu(y))}$, the optimality conditions can be written a

$$
\begin{aligned}
& m^{\prime}(\Lambda(y)) \cdot f(\mu(y), y)=A(\mu(y)), \\
& q(\Lambda(y)) \cdot f_{x}(\mu(x), y)=A^{\prime}(\mu(y)) .
\end{aligned}
$$

These equations are identical to first-order conditions (6) and (7) of the decentralized economy with appropriate reinterpretation of the variables.

To establish that the solution to this program is identical to the solution of the decentralized economy, focus on the case of positive sorting (the alternative case follows analogous steps). The planner's boundary conditions are the following: at the upper bound, assortative matching means that $\mu(\bar{y})=\bar{x}$; at the lower bound, observe that it is never optimal to assign lower types if higher types have matching probability zero. Therefore, $\Lambda(y)=0$ or $\Lambda(y)=\infty$ only at $y=y_{0}$. Moreover, obviously $y_{0} \geq \underline{y}$ and $\mu\left(y_{0}\right) \geq \underline{x}$. Therefore, the planners' problem has the same boundary conditions as the decentralized equilibrium. In the proof of existence (Proposition 2), we showed that under $n$-rootsupermodularity for $n=(1-\bar{a})^{-1}$ any solution of these first-order conditions and the boundary solutions constitutes an equilibrium when integrated up to the corresponding distributions $(G, H)$.

Finally, we sketch why the planner's solution is positive assortative if $f$ is $n$-root-supermodular with $n=(1-\bar{a})^{-1}$. Assume that the planner's solution locally yields a differentiable assignment: on some subset of $\mathcal{X} \times \mathcal{Y}$, the distribution $H$ fulfills $H_{\mathcal{X Y}}(\mu(y), y)=B(x)$ for some function $\mu$ that is differentiable. Optimality still requires that $\mu^{\prime}$ satisfies (38) and associated optimality conditions (39) and (40). Yet to maximize the Hamiltonian, the second-order condition must be satisfied: (39) and (40) are identical to (6) and (7) under appropriate relabeling of variables, and the second-order condition therefore reduces to (14), which requires positive sorting. This rules out locally decreasing assignments. A tedious proof that extends this logic globally is available from the authors.

Q.E.D.

ProOf OF THE EQuilibriUm PRICE SCHEdule: In a differentiable assortative equilibrium with price function $p(y)$, assignment function $\mu(y)$, and queue length $\Lambda(y)$ [formally $\Lambda(y, p(y))$ ], the equilibrium buyer utility $U(\mu(y))=q(\Lambda(y))[f(\mu(y), y)-p(y)]$ can be totally differentiated to get

$$
U^{\prime} \mu^{\prime}=\Lambda^{\prime} q^{\prime}[f-p]+q\left(f_{x} \mu^{\prime}+f_{y}-p^{\prime}\right),
$$


where we suppressed all arguments. Note further that firms' equilibrium profits can be recovered by substituting (6) into (5), yielding $\left[m-\Lambda m^{\prime}\right] f$. Equating this to the definition of expected profits as trading probability times price (i.e., $m p$ ), we obtain the price schedule $p(y)$ along the equilibrium path as $p=\left[1-\Lambda m^{\prime} / m\right] f=\left[1-\eta_{m}\right] f$. Substituting this and (6) into (41), we get after canceling terms that $0=q^{\prime} \eta_{m} \Lambda^{\prime} f+q\left[f_{y}-p^{\prime}\right]$. We can solve this for $p^{\prime}$ : use (11) to substitute out $\Lambda^{\prime} f$ and use the fact that $a=m^{\prime} q^{\prime} /\left(m^{\prime \prime} q\right)$ to get, after rearranging, that $p^{\prime}=f_{y}+a\left[f_{x} \mu^{\prime}\left(m / \Lambda-m^{\prime}\right) \eta_{m} / m^{\prime}-\left(1-\eta_{m}\right) f_{y}\right]$. Since $\left[m / \Lambda-m^{\prime}\right] \eta_{m} / m^{\prime}=1-\eta_{m}$, we obtain (17).

Q.E.D.

\section{REFERENCES}

ACEMOGLU, D., AND R. SHimer (1999a): “Efficient Unemployment Insurance,” Journal of Political Economy, 107, 893-928. [545,557]

(1999b): "Holdups and Efficiency With Search Frictions," International Economic Review, 40, 827-849. [543,545,546,557]

BECKER, G. S. (1973): “A Theory of Marriage: Part I,” Journal of Political Economy, 81, 813-846. [539,540,552,555,556,559-562]

BurdetT, K., S. SHI, AND R. WRIGHT (2001): "Pricing and Matching With Frictions," Journal of Political Economy, 109, 1060-1085. [557]

ButTers, G. R. (1977): "Equilibrium Distributions of Sales and Advertising Prices," Review of Economic Studies, 44, 465-491. [544]

DelacRoiX, A., AND S. SHI (2006): “Directed Search on the Job and the Wage Ladder," International Economic Review, 47, 651-699. [558]

EECKHOUT, J., AND K. PHILIPP (2010): "Sorting versus Screening: Search Frictions and Competing Mechanisms," Journal of Economic Theory (forthcoming). [558]

GALE, D. (1986): "Bargaining and Competition, Part I: Characterization," Econometrica, 54, 785-806. [561]

GALENIANOS, M., AND P. KiRCHER (2009): "Directed Search With Multiple Job Applications," Journal of Economic Theory, 114, 445-471. [558]

HICKS, J. R. (1932): The Theory of Wages. London: Macmillan. [550]

Hosios, A. (1990): "On the Efficiency of Matching and Related Models of Search and Unemployment," Review of Economic Studies, 57, 279-298. [543,557,562]

Kantorovich, L. V. (1958): “On the Translocation of Masses," Management Science, 5, 1-4. [553]

KIRCHER, P. (2009): “Efficiency of Simultaneous Search,” Journal of Political Economy, 117, 861-913. [558]

KiYOTAKi, N., AND R. Wright (1993): "A Search-Theoretic Approach to Monetary Economics," American Economic Review, 83, 63-77. [545]

LAUERmanN, S. (2007): "Dynamic Matching and Bargaining Games: A General Approach," Mimeo, University of Michigan. [561]

Mas-Colell, A. (1984): “On a Theorem by Schmeidler," Journal of Mathematical Economics, 13, 201-206. [545]

MCAFEE, R. P. (1993): “Mechanism Design by Competing Sellers,” Econometrica, 61, 1281-1312. [546,558]

MenzIO, G. (2007): “A Theory of Partially Directed Search,” Journal of Political Economy, 115, 748-769. [554]

MoEN, E. (1997): “Competitive Search Equilibrium,” Journal of Political Economy, 105, 385-411. $[543,557]$

MorTensen, D., AND R. Wright (2002): “Competitive Pricing and Efficiency in Search Equilibrium," International Economic Review, 43, 1-20. [558] 
Peters, M. (1991): "Ex ante Pricing in Matching Games: Non Steady States,” Econometrica, 59, 1425-1454. [540,544-546,557]

(1997a): “A Competitive Distribution of Auctions," The Review of Economic Studies, 64, 97-123. [540,546,558]

(1997b): "On the Equivalence of Walrasian and Non-Walrasian Equilibria in Contract Markets: The Case of Complete Contracts," The Review of Economic Studies, 64, 241-264. [557]

(2000): "Limits of Exact Equilibria for Capacity Constrained Sellers With Costly Search," Journal of Economic Theory, 95, 139-168. [545,546,557]

(2007): "Unobservable Heterogeneity in Directed Search," Mimeo, UBC. [557]

Rosen, S. (1974): "Hedonic Prices and Implicit Markets: Product Differentiation in Pure Competition," Journal of Political Economy, 82, 34-55. [539,540,546,560]

(2002): "Markets and Diversity," American Economic Review, 92, 1-15. [540]

RUBINSTEIN, A., AND A. WOLINSKY (1985): "Equilibrium in a Market With Sequential Bargaining," Econometrica, 53, 1133-1150. [561]

SHI, S. (2001): "Frictional Assignment. 1. Efficiency," Journal of Economic Theory, 98, 232-260. [541,543,544,549,552,557,558]

(2002): "A Directed Search Model of Inequality With Heterogeneous Skills and SkillBiased Technology," The Review of Economic Studies, 69, 467-491. [558]

SHIMER, R. (2005): "The, Assignment of Workers to Jobs in an Economy With Coordination Frictions," Journal of Political Economy, 113, 996-1025. [544,546,558]

Shimer, R., AND L. SMith (2000): "Assortative Matching and Search,” Econometrica, 68, 343-369. [539,542,559,560]

SMITH, L. (2006): “The Marriage Model With Search Frictions," Journal of Political Economy, 114, 1124-1144. [542]

Dept. of Economics, University of Pennsylvania, 3718 Locust Walk, Philadelphia, PA 19140, U.S.A. and UPF Barcelona, ICREA, and GSE; eeckhout@ssc. upenn.edu

$$
\text { and }
$$

Dept. of Economics, University of Pennsylvania, 3718 Locust Walk, Philadelphia, PA 19140, U.S.A. and University of Oxford, Oxford, U.K.; kircher@econ. upenn.edu.

Manuscript received May, 2008; final revision received October, 2009. 